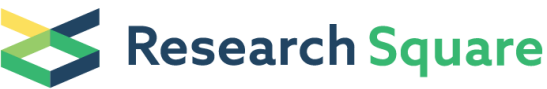 \\ Preprints are preliminary reports that have not undergone peer review. \\ They should not be considered conclusive, used to inform clinical practice, or referenced by the media as validated information.
}

\section{The Blursday Database: Individuals' Temporalities in Covid Times}

\section{Maximilien Chaumon}

Institut du Cerveau, ICM, Inserm U1127, CNRS UMR 7225, Sorbonne Université, Centre MEG-EEG, Centre de Neurolmagerie Recherche (CENIR)

\section{Pier-Alexandre Rioux}

École de psychologie, Université Laval

\section{Sophie Herbst}

Cognitive Neuroimaging Unit, INSERM, CEA, CNRS, Université Paris-Saclay, NeuroSpin

\section{Ignacio Spiousas}

Universidad Nacional de Quilmes (CONICET) https://orcid.org/0000-0002-0283-4696

\section{Sebastian Kübel}

Max Planck Institute for the Study of Crime, Security and Law; Institute for Frontier Areas of Psychology and Mental Health https://orcid.org/0000-0001-7072-9508

\section{Elisa Gallego Hiroyasu}

Department of Life Sciences, The University of Tokyo https://orcid.org/0000-0003-3014-9183

\section{Serife Runyun}

Department of Psychology and Center for Translational Medicine, Koç University

\section{Luigi Micillo}

Department of General Psychology, University of Padova, Italy

\section{Vassilis Thanopoulos}

Multisensory and Temporal Processing Laboratory (MultiTimeLab), Department of Psychology,

Panteion University of Social and Political Sciences

\section{Esteban Mendoza-Durán}

École de psychologie, Université Laval, https://orcid.org/0000-0002-1524-4273

\section{Anna Wagelmans}

Cognitive Neuroimaging Unit, INSERM, CEA, CNRS, Université Paris-Saclay, NeuroSpin

\section{Ramya Mudumba}

Department of Cognitive science, Indian Institute of Technology Kanpur

\section{Rania Tachmatzidou}

Multisensory and Temporal Processing Laboratory (MultiTimeLab), Department of Psychology, Panteion University of Social and Political Sciences

\section{Nicola Cellini}

University of Padua https://orcid.org/0000-0003-0306-4408 
https://orcid.org/0000-0003-3618-9768

\section{Anne Giersch}

Université de Strasbourg, Unité mixte INSERM U1114, Département de Psychiatrie, Hôpital civil

\section{Simon Grondin}

Universite Laval

\section{Claude Gronfier}

Neurocampus, Lyon Neuroscience Research Center, INSERM U1028, CNRS UMR5292, University Lyon 1

\section{Federico Alvarez Igarzábal}

Institute for Frontier Areas of Psychology and Mental Health

\section{André Klarsfeld}

ESPCI, Laboratoire Plasticité du Cerveau, UMR 8249, CNRS

\section{Ljubica Jovanovic}

University of Nottingham https://orcid.org/0000-0003-0977-3379

\section{Rodrigo Laje}

Department of Science and Technology, University of Quilmes; CONICET (Consejo Nacional de Investigaciones Científicas y Técnicas) https://orcid.org/0000-0003-1481-303X

\section{Elisa Lannelongue}

Cognitive Neuroimaging Unit, INSERM, CEA, CNRS, Université Paris-Saclay, NeuroSpin

\section{Giovanna Mioni}

University of Padova https://orcid.org/0000-0002-1212-4591

\section{Cyril Nicolaï}

Cognitive Neuroimaging Unit, INSERM, CEA, CNRS, Université Paris-Saclay, NeuroSpin; Centre de Recherches Interdisciplinaires

\section{Narayanan Srinivasan}

Indian Institute of Technology Kanpur

\section{Shogo Sugiyama}

Department of Life Sciences, The University of Tokyo

\section{Marc Wittmann}

Institut für Grenzgebiete der Psychology und Psychohygiene https://orcid.org/0000-0002-4483-7334

\section{Yuko Yotsumoto}

Department of Life Sciences, The University of Tokyo

\section{Argiro Vatakis}

Multisensory and Temporal Processing Lab (MultiTimeLab), Department of Psychology, Panteion University of Social and Political Sciences, Athens

\section{Fuat Balci}

Department of Biological Sciences, University of Manitoba; Department of Psychology and Center for Translational Medicine, Koç University

\section{Virginie van Wassenhove ( $\square$ Virginie.van.Wassenhove@gmail.com )}

CEA NeuroSpin, Université Paris-Saclay https://orcid.org/0000-0002-2569-5502 


\section{Resource}

\section{Keywords:}

Posted Date: December 1st, 2021

DOI: https://doi.org/10.21203/rs.3.rs-1098637/v1

License: (c) (i) This work is licensed under a Creative Commons Attribution 4.0 International License. Read Full License

Version of Record: A version of this preprint was published at Nature Human Behaviour on August 15th, 2022. See the published version at https://doi.org/10.1038/s41562-022-01419-2. 


\section{Abstract}

The Covid-19 pandemic and associated lockdowns triggered worldwide changes in the daily routines of human experience. The Blursday database provides measures of subjective time and related processes from more than 2,800 participants (over 9 countries) tested on 14 questionnaires and 15 behavioral tasks during the Covid-19 pandemic. The easy-to-process database and all data collection tools are made fully accessible to researchers interested in studying the effects of social isolation on temporal information processing, time perspective, decision-making, sleep, metacognition, attention, memory, self-perception, and mindfulness. Blursday also includes vital quantitative statistics such as sleep patterns, personality traits, psychological well-being, and lockdown indices. Herein, we exemplify the use of the database with novel quantitative insights on the effects of lockdown (stringency, mobility) and subjective confinement on time perception (duration, passage of time, temporal distances). We show that new discoveries are possible as illustrated by an inter-individual central tendency effect in retrospective duration estimation.

\section{Introduction}

Subjective time is one of the most malleable aspects of personal experience, which can be altered by many exogenous factors (e.g., physical features of the environment, social interactions) as well as endogenous psychological and physiological states (e.g., arousal, attention, valence, febricity, circadian rhythms). An altered sense of time can be indicative of individuals' well-being (Holman \& Grisham, 2020) and misguide individuals' decisions and judgments (Wittmann \& Paulus, 2008). At a historically global scale, the Covid-19 pandemic and associated lockdowns and state of emergency measures deeply altered the living conditions, social interactions, and the psychological, physiological and economic wellbeing of the entire human population. Although the effects of the pandemic and of the lockdown measures on mental health have already started to be documented extensively (e.g., WHO; https://www.covidminds.org (Yamada et al., 2021)), how an altered sense of time accompanies, and perhaps even accounts for some of these major changes, has not been examined in a comprehensive and systematic manner.

The Blursday project tackles this challenge and provides an unprecedentedly rich and comprehensive dataset for characterizing temporalities of more than 2800 participants collected in 9 different countries (4 continents) during the peak of the lockdown periods. The uniqueness of the Blursday database is its large number of widely utilized behavioral tasks (performance measures) combined with questionnaires (self-reports), demographics and subjective confinement trackers (state measures). The database includes participants tracked longitudinally in and out of lockdown (from 2020 on) together with control groups of naïve participants tested outside of Covid-19 lockdowns (from 2021 on). The study has thus been designed to serve as a rich empirical benchmark to investigate how temporalities and related processes changed during the historical episode of the Covid -19 pandemic.

There are several reasons why time perception is central beyond the observation that disorientations in time were a phenomenologically vivid and widely shared experience during lockdown. First, all animals 
keep track of time at multiple time scales regulated by physiological clocks e.g., circadian rhythms, interval timing, motor timing (Buonomano, 2007; Laje et al., 2018). Second, interval timing in the secondsto-minutes range is most malleable because it holds strong ties with domain-general cognition including attention, memory, or decision-making (Balci, Meck, et al., 2009). Timekeeping systems also interact with each other: interval and motor timing fluctuate with circadian rhythms, which is essential for the adaptiveness of other cognitive functions and related behaviors (Schmidt et al., 2007). In turn, the accuracy and precision of temporal representations and behaviors heavily influence decisionmaking (Balcı et al., 2011).

Temporal phenomenologies are thus crucial for our understanding of how cognition generally, and timekeeping specifically, have been affected by the altered lifestyles and profound routine changes during the lockdown periods. Recent studies started showing changes in sleep patterns (Blume et al., 2020; Cellini et al., 2020), levels of physical activity (Marelli et al., 2021) and increase in depression, anxiety, and fear across countries (Dean et al., 2021; Lieberoth et al., 2021; Micillo et al., 2021; Yamada et al., 2021). In the international study COVIDiSTRESS (Yamada et al., 2021), surveys and self-report questionnaires have focused on perceived distress. Covid-19 lockdowns have been suggested to negatively affect executive functions, attention, and anecdotally temporal orientation through the self-reporting of forgotten dates during that period (Fiorenzato et al., 2021). Based on established relations between these factors and interval timing, one would expect our time keeping ability to be dramatically affected by lockdowns.

Self-reports on the passage of time during lockdown have been promptly published. In an Italian study (Cellini et al., 2020), surveyed participants reported pronounced problems with keeping track of the hours and days, and they also reported an expansion of subjective duration which was associated with a sense of boredom. In French surveys, participants answered the question: "What are your feelings about the speed of passage time?" (Droit-Volet et al., 2020, 2021; Martinelli et al., 2020). The question was asked in reference to the individuals' autobiographical recall of their experienced passage of time before the lockdown, then asked three times during lockdown in reference to the "now", to "yesterday", and to "one week ago". A trend towards the passage of time feeling slower during, as compared to before the lockdown was reported for all temporal scales. The best predictors of the experience of the passage of time were boredom and sadness, which also contributed most to the well-being of French participants during and after lockdown. In the UK surveys (Ogden, 2020, 2021), participants reported a significant distortion of the passage of time in both directions, attributable to stress but also to age, task load, and one's satisfaction with the experienced social interactions. A comparable number of participants felt that the last day and the last week had either passed more quickly or more slowly than usual; additionally, the older and less socially satisfied British individuals were, the slower time seemed to pass during both UK lockdowns. In a Uruguayan study (Loose et al., 2021), which assessed the experience of university students, the authors reported an association between psychological distress due to the Covid-19 restrictions and the feeling of a slower passage of time, a blurred sense of time (not knowing what time or day it is), and more boredom. In a longitudinal Brazilian study (Cravo et al., 2021), participants initially perceived an expansion of time which steadily decreased over the course of the following weeks. These surveys confirmed self-reported temporal distortions during lockdown but do not converge on their 
underlying explanatory causes. Several experimental limitations include the absence of controls, which prevents assigning a causal role of lockdown to temporal distortions, and the possibility that crosscultural factors and differences in the stringency of the lockdown measures played a crucial role in the different trends observed across countries. All studies used questionnaires and ratings, and none included psychometric tasks. The Blursday database herein includes a large and diverse battery of questionnaires, tasks and tests across cultures, a longitudinal assessment of these factors within individuals and, importantly, a control population, naive to all tasks, tested outside of lockdown.

We provide a comprehensive dataset that captures subjective time and timing behaviors of a large number of participants on nearly all aspects of temporal information processing (duration perception and production, spontaneous tapping, synchronization, implicit timing) together with measures of working memory, decision-making, self-perception, metacognition, sleep patterns, personality traits, and wellbeing. The Blursday database provides an extensive benchmark for future studies that would incorporate new control data although the current database already contains some control data. We make all tools available online for researchers wishing to test participants post-lockdown using the same approach or parts of it. We fully describe the database and importantly, provide examples illustrating its potential use based on a few fundamental analyses providing novel quantitative insights on time perception during this historical period.

\section{Methods}

\section{Participants}

As of November 8th 2021, a total of 2,840 participants signed up for the online "Time Social Distancing" study in 12 countries (Argentina, Canada, Colombia, France, Germany, Greece, India, Italy, Japan, Turkey, United Kingdom, United States of America). The attrition rate was predictably very important in the course of the longitudinal study: 439 participants finished all tasks in the first session (S1; Table 1 and Supp. Fig. 1) conducted during the first lockdown; 200 participants finished the second session (S2) and 244 the third session (S3) which took place outside the initial lockdown confinement and about 2 weeks and 3 months after it, respectively. In some countries (France and Italy) a shortened fourth session was conducted (S4) on the same participants during their second lockdown with 275 participants. At least a year later, starting in May 2021, as restrictions started to be lifted in some countries, a control pool of naïve participants was recruited in each country. As of November 8th 2021, 243 participants completed the control session. This control population was tested on the full set of questionnaires and tasks originally tested in S1. As of November 8th 2021, these tests are finished in some countries (e.g. France, Japan, Italy, Germany), ongoing (e.g., Greece, Turkey) or planned (e.g., Argentina) in others.

In all sessions, participants reporting drug usage and psychiatric disorders were a priori excluded from data collection; some of the included questionnaires otherwise allow for an evaluation of depression, stress, anxiety and attenuated symptoms of psychosis. Data from Colombia, UK, and USA were too few to be included and were a priori discarded from most analyses (although made available in the 
database). Due to the exceptional nature and speed of change in governmental policies, experiments started during the first lockdown or state-of-emergency of each country in 2020 (Fig. 1) and continued longitudinally at a different pace according to local policies. We report in Fig. 1 the full demographics of the database during the experiments along with lockdown dates and general timelines of the study.

\section{Ethics}

All participants were provided with full instructions and signed an online consent form following the Declaration of Helsinki (2018) and the ruling of Ethical committees. Participants were provided with a contact email if they had any questions before proceeding. The approval to run the study internationally was obtained from the University Paris-Saclay (CER-Paris-Saclay-2020-020). We also sought local approval for each country: Comité de Etica de la Universidad Nacional de Quilmes CE-UNQ No 2/2020 (Argentina); Université Laval, 2020-114 / 14-04-2020 (Canada); Institute for Frontier Areas of Psychology and Mental Health, Freiburg, IGPP_2020_01 (Germany, Switzerland, Austria); Ethical Committee for the Psychological Research of the University of Padova (Italy); Institutional Ethics Committee, Indian Institute

of Technology Kanpur, IITK/IEC/2019-20/18-Apr-20/I (India); The Institutional Review Board of the University of Tokyo, \#705 (Japan): UCLA Office of the Human Research Protection Program, IRB\#20000612 (USA); Koç University, 2020.113.IRB3.053 (Turkey).

\section{Data Acquisition Procedure}

We used the Gorilla Experiment Builder (www.gorilla.sc) to build and host our study (Anwyl-Irvine et al., 2019) in several languages and countries. The original project was designed in English. French, Japanese, Italian, Greek, Portuguese, German, Spanish, and Turkish were cloned from the original English templates, translated, and beta-tested by the local teams, and eventually adapted to the needs or cultural specificities of the country. All questionnaires and tasks are freely accessible in English (and other languages, see below) under the Gorilla Open Materials Attribution-NonCommercial Research-Only licensing: https://app.gorilla.sc/openmaterials/278377.

In most countries, participants were recruited by means of general advertisement using institutional newsletters and/or outside the institution through social media channels. In Japan, participants were recruited through an agency or online (half of the participants for the control session); all participants were given monetary rewards for completing each session. In France, participants in the control session were given an option to receive a small compensation for their participation and $\sim 43 \%$ of them chose so (80 out of 184 participants). In Turkey and Greece, a group of participants was recruited through classes and compensated with bonus course credits.

General information was provided in different languages and updated over time for each country on a specific web page (https://brainthemind.com/covid19/) as well as locally in printed form (https://osf.io/359qm/). When participants connected to the protocol website, they were first provided 
with general information about the study and asked to provide their consent. Then, they were invited to create an anonymized public identification, which they kept for the rest of the study. Participants could leave the website and come back where they stopped at any time. They were free to stop the experiment when they wanted to. Any technical issue, bug, or any problem participants would have was handled by email.

\section{Protocol}

The full experimental protocol consisted in three to four longitudinal sessions (S1, S2, S3, S4) and one control session (SC, new participants). In all sessions, participants went first through a series of questionnaires administered in a random order across participants, which they had to take once per session for the majority of them. After the series of questionnaires, they entered in a series of diverse behavioral tasks presented in pseudo-random order (latin-square design) across participants. Each task was presented up to three times within a session. In the course of the study, the number of runs was reduced to lighten up the requirements of the study. A general insight on the full session is described in Supp. Fig.1 and Table 1. Both provide a comprehensive description of the content of each session. A detailed description of questionnaires and tasks used in the study is provided below.

\section{Questionnaires}

We included an extensive number of questionnaires that have been (cross-)validated in different languages and in several countries as well as designed new ones. Answering the first series of questionnaires took about an hour. We designed a Confinement Tracker questionnaire and an Isolation Questionnaire adapted to the circumstances to provide basic information on the state of lockdown (Supp. Mat). We included the UCLA Loneliness Scale (Russell, 1996; Russell et al., 1980) which provides several metrics of self-reported loneliness. The clinically-oriented Hospital Anxiety and Depression Scale or HADS (Crawford et al., 2001) provides reliable measures of the state of anxiety and depression of participants. The PQ16 (Ising et al., 2012) was used to screen participants' attenuated symptoms of psychosis. Mindfulness was assessed using the Freiburg Mindfulness Inventory or FMI (Walach et al., 2006). Circadian preferences and sleep disturbances were assessed using the Morningness-Eveningness Questionaire reduced version or rMEQ (Randler, 2013), the ultra-short version of the Munich Chronotype Questionnaire $\mu \mathrm{MCTQ}$ (Ghotbi et al., 2020), as well as monthly and weekly versions of the Pittsburgh Sleep Quality Index or PSQI (Buysse et al., 1991; Smyth, 1999), and a daily sleep quality questionnaire (Supp. Mat). The general personality traits of participants were assessed using the Big Five Inventory or BFI-10 (Rammstedt, 2007; Rammstedt \& John, 2007). The Zimbardo Time Perspective Inventory or ZTPI (Zimbardo, 1990; Zimbardo \& Boyd, 2015) provides a general assessment of the individual's temporal orientation trait (Sircova et al., 2014). The attentional orientation trait of participants was assessed using the Attentional Style Questionnaire or ASQ (Kraft et al., 2019; Van Calster et al., 2018). 
Table 1: Overview of tasks and questionnaires. Sessions 1 and 4 took place during the first and the second lockdown, respectively. Sessions 2 and 3 were set at least 2 weeks and 3 months after the first lockdown. Thus, sessions 1, 2, 3, and 4 tested the same set of participants longitudinally in and out lockdown. The Control session tested a group of naive participants on the same set of questionnaires and tasks as those tested in Session 1 (during the first lockdown). A detailed description of Session 1 is provided in Supp. Fig.1.

\begin{tabular}{|c|c|c|c|c|c|}
\hline & $\begin{array}{l}\text { SESSION } 1 \\
\text { confinement }\end{array}$ & $\begin{array}{c}\text { SESSION 2 } \\
\text { at least } 2 \text { weeks } \\
\text { post-confinement }\end{array}$ & $\begin{array}{c}\text { SESSION 3 } \\
\text { at least } 3 \text { months } \\
\text { post-confinement }\end{array}$ & $\begin{array}{c}\text { SESSION } 4 \\
\text { Confinement } 2 \\
\text { France and ltaly only }\end{array}$ & $\begin{array}{c}\text { CONTROL } \\
\text { at least } 1 \text { year } \\
\text { post-confinement }\end{array}$ \\
\hline 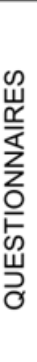 & $\begin{array}{ll}: & \text { Confinement status } \\
\text { Demographics } \\
\text { Environment and social connectivity } \\
\text { (UCLA) } \\
\text { Isolation questionnaire } \\
\text { Morningness-Eveningness Chronotype } \\
\text { (rMEQ) } \\
\text { jMCTO } \\
\text { Daily sleep quality } \\
: \quad \text { Zimbardo Time Perspective Inventory } \\
\text { Hospital Anxiety and Depression Scale } \\
\text { (HADS) } \\
\text { Pittsburgh Sleep Quality Index (PSQI) } \\
: \quad \begin{array}{l}\text { Big Five Inventory (BFI-10) } \\
:\end{array} \text { Attentional Style Questionnaire (ASQ) } \\
\text { Self-Perception (PQ16) } \\
\text { FFA }\end{array}$ & $\begin{array}{ll}: & \text { Confinement status } \\
\text { UCLA } & \text { Isolation questionnaire } \\
: & \text { HADS } \\
: & \text { PSQI } \\
: & \text { HMCTQ } \\
\text { Dalily sleep quality } & \\
\text { FFA }\end{array}$ & $\begin{array}{ll}: & \text { Confinement status } \\
: & \text { UCLA } \\
: \quad & \text { Isolation questionnaire } \\
\text { HADS } \\
: \quad \text { PSQI } \\
\text { HMCTQ } \\
: \quad \text { Daily sleep quality } \\
\text { PQ16 } \\
\text { FFA }\end{array}$ & 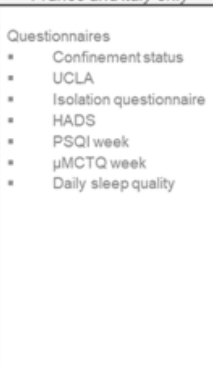 & 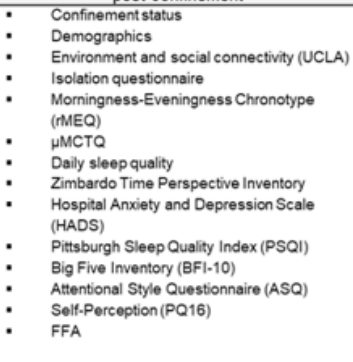 \\
\hline $\begin{array}{l}0 \\
\frac{1}{0} \\
\frac{1}{1}\end{array}$ & $\begin{array}{ll}: & \text { Retrospective Duration } \\
: & \text { Passage of Time } \\
: & \text { Tempoctive Temporal distance } \\
\text { - } & \text { Fluency tandmarks (Phonemic, Semantic, } \\
& \text { Time Semantic, Past, Future) } \\
: & \text { Duration production and metacognition } \\
: & \text { Tapping and synchronization } \\
\text { - } & \text { Delay-Discounting } \\
\text { n-back Working memory, prospective } \\
\text { duration and passage of time } \\
\text { Counting task and duration estimation } \\
\text { Implicit timing / foreperiod } \\
\quad \text { Self Preference }\end{array}$ & $\begin{array}{ll}\text { : } & \text { Retrospective Duration } \\
\text { : } & \text { Passage of Time } \\
\text { - } & \text { Temjective Temporal landmark distance } \\
\text { - Fluency tasks (Phonemic, Semantic, } \\
\text { - Time Semantic, Past, Future) } \\
\text { Duration production and } \\
\text { metacognition } \\
\text { Tapping and synchronization } \\
\text { Delay-Discounting } \\
\text { n-back Working memory, prospective } \\
\text { duration and passage of time } \\
\text { Counting task and duration estimation } \\
\text { Implicit timing / foreperiod } \\
\text { Self Preference }\end{array}$ & $\begin{array}{ll} & \text { Retrospective Duration } \\
: & \text { Passage of Time } \\
\text { Subjective Temporal distance } & \text { Temporal landmark } \\
\text { - Fluency tasks (Phonemic, } \\
\text { Semantic, Time Semantic, Past, } \\
\text { Future) } \\
\text { Duration production and } \\
\text { metacognition } \\
\text { Tapping and synchronization } \\
\text { Delay-Discounting } \\
\text { n-back Working memory. } \\
\text { prospective duration and passage } \\
\text { of time } \\
\text { Counting task and duration } \\
\text { estimation (only in Sessions 1-3) } \\
\text { Implicit timing / foreperiod } \\
\text { Self Preference }\end{array}$ & $\begin{array}{ll}\text { Subjective Temporal } \\
\text { distance }\end{array}$ & $\begin{array}{ll} & \text { Retrospective Duration } \\
\text { Passage of Time } \\
\text { Subjective Temporal distance } \\
\text { Temporal landmark } \\
\text { Fluency tasks (Phonemic, Semantic, Time } \\
\text { Semantic, Past, Future) } \\
\text { Duration production and metacognition } \\
\text { : Tapping and synchronization } \\
\text { Delay-Discounting } \\
\text { n-back Working memory, prospective } \\
\text { duration and passage of time } \\
\text { Implicit timing / foreperiod } \\
\text { Self Preference }\end{array}$ \\
\hline
\end{tabular}

\section{Tasks}

\section{Retrospective Duration}

The vast majority of studies in time perception use prospective timing tasks in which participants know beforehand they will be asked to estimate the duration of an upcoming event or stimulus (Block et al., 2018). While helpful (see below for prospective duration tasks), this paradigm also falls short of capturing temporal judgments that are commonly made retrospectively in daily life. Retrospective temporal judgments require individuals to make an estimate of elapsed time since a past event or during an activity that just happened without them knowing a priori they will have to time (Block et al., 2018; Grondin \& Plourde, 2007; Hicks, 1992; Zakay \& Block, 1997). Cognitive components (e.g., attention and memory) are considered to be differentially involved during retrospective $v s$. prospective timing (Block et al., 2018) with retrospective duration estimates assumed to engage episodic memory processes. In the Blursday project, we included retrospective duration estimations (Supp. Fig. 2) at several moments after a series of questionnaires or after specific tasks. Herein, we report the first retrospective duration estimate participants had to make in the study, which followed a series of initial questionnaires and thus spanned a scale of minutes to hours. The outcomes are included in the Results section and illustrated in Figure 4. 


\section{Passage of Time Judgments}

Passage of time judgments can be used to estimate the subjective feeling that time passes otherwise commonly referred to as the "flow of time" (Thönes \& Stocker, 2019; Wearden, 2015). In this study, passage of time judgments were either implemented as Visual Analog Scales (VAS) ranging from "very slow" to "very fast" or as Likert scales offering a categorical choice between: "very slow", "slow", "normal", "fast", and "very fast". Like for retrospective duration estimates, we used passage of time judgments after several tasks during the study by asking participants to report how fast time felt in a given lapse of time (e.g., Supp. Fig. 5). Herein, we report the passage of time judgments that were estimated using a VAS and over the scale of the "last few days" (Supp. Fig. 2) in the Results section.

\section{Temporal landmarks and event recording}

By analogy to spatial landmarks, temporal landmarks are salient events that have been time stamped in memory. For instance, one's birthday tends to be an important landmark. One way to assess the existence of temporal landmarks is to evaluate the speed (response times) and ease (error rate) with which one answers a question about a point in time. Chronometry and performance can be driven by the psychological distance of that point in time from the operative landmark in one's temporal cognitive map. Temporal landmarks can be culturally and autobiographically idiosyncratic. For instance, when participants are asked to answer as fast and as accurately as possible "What day is it?", the closer a day is to a cultural temporal landmark (e.g. Sunday in Catholicism or Shabbat in Judaism), the faster the responses and the lower the error rates (Koriat et al., 1976; Koriat \& Fischhoff, 1974). In this study, we prompted participants at various times with the question "What day is it today?", as well as asked them to report an important event for them on that same day (Supp. Fig. 2). The distribution of the collected responses times during lockdown in all participating countries are illustrated in Fig. $\mathbf{2 d}$.

\section{Subjective Temporal distance}

An estimation of subjective temporal distance consists in asking participants to estimate how far away an event feels for them. Subjective temporal distances involve episodic memory processes and the abstraction of temporal relations between events (Friedman, 1993; Liberman \& Trope, 2014). Herein, we asked participants to use a VAS to report how far away their first day of lockdown felt with respect to the moment at which they were asked this question (i.e., the present). This subjective temporal distance provides a subjective measure of elapsed time at the scale of days to weeks and months as recalled by the participant (Supp. Fig. 2). We also assessed participants' subjective distance to a week and a month ahead, to test their future orientation. Although subjective distances may be related to the actual passage of time, people may feel more or less close to a past event regardless of its actual temporal distance (Ross \& Wilson, 2002). The outcomes of these ratings are included in the Results section and illustrated in Fig. 5.

\section{Fluency tasks: Semantic, Phonemic, and Time scales}


Verbal fluency tasks involve reporting as many words as possible within an imparted lapse of time, based on phonemic or semantic criteria (Henry \& Crawford, 2004). These tasks were originally developed for neurolinguistic and cognitive assessments. For instance, a semantic fluency task consists of asking participants to report as many animals as possible in 60 seconds (s); this was the semantic fluency task included in our study (Supp. Fig. 3). Similarly, the phonemic fluency task consisted here of reporting as many words as possible starting with the letter ' $P$ ' in $60 \mathrm{~s}$. In addition to classic verbal fluency tasks, we included past and future event fluency tasks to assess the accessibility of mental representations of life events that participants experienced in the past or that they plan for the future (D'Argembeau et al., 2010). These fluency tasks took the form of a question "Write as many events as possible that occurred last week/moth/year]" for past fluency or "that will happen next [week/month/year" for the future fluency tasks (Supp. Fig. 3). Hence, these fluency tasks tested the scales at which the fluency was assessed, namely over a week, a month, or a year. An additional semantic fluency task inquiring about associations with the word "time" was tested by simply asking participants to report as many spontaneous associations as possible they had with this word. All fluency tasks in the Blursday database were $60 \mathrm{~s}$ long and the number of collected items was unlimited.

\section{Prospective duration estimation while counting up or down}

When participants prospectively estimate a lapse of time, both attention and working memory influence their duration estimates. The demonstration of this influence is often based on a dual-task paradigm in which a participant is asked to perform both a temporal and a nontemporal task. Several nontemporal tasks have been used to show the impact of attention or working memory on prospective judgments of time (Brown, 1997; Grondin \& Macar, 1992; Macar et al., 1994). Amongst these tasks, there is the possibility to ask participants to perform a counting task (Brown, 1997), a strategy that is adopted in the present investigation. Herein, participants were prompted with a prime number and asked to count up (addition) or down (subtraction) in steps of 3 or 7 as a way to control the difficulty of the task (Supp. Fig. 4). Following a trial, participants were asked to report the number they reached as well as the amount of time spent doing the task (which could be, unbeknownst to participants, either $12 \mathrm{~s}$ or $24 \mathrm{~s}$ ).

\section{Prospective duration estimation while performing an n-back task}

Level of processing in working memory lengthened temporal production presumably by slowing down temporal integration (Fortin \& Breton, 1995). One means to further explore the influence of working memory on time estimation is to use a parametrically variable n-back task in which a sequence of letters is displayed on the screen and participants decide on a trial-by-trial basis whether the displayed letter is identical to the previous one $(n=1)$ or to the one two letters before it $(n=2)$ and so on. It has recently been shown that increasing the working memory load (increasing the $n$ ) may proportionally shorten the prospective estimation of duration whereas paying attention to time may lengthen it in an additive fashion (Polti et al., 2018). In this study, we asked participants to perform an $n$-back task ( $n=1$ or $n=3$ ) and to report how long the trial was (in minutes:seconds) as well as how fast time felt on a Likert scale. Unbeknownst to participants, a trial could last 45 s or 90 s (Supp. Fig. 5). 


\section{Duration production and metacognition}

Duration production is another prospective timing task which consists of asking participants to estimate a time interval using overt motor behavior. Herein, participants were asked to produce $3.6 \mathrm{~s}$ by pressing the spacebar to initiate their time estimation and, once they considered that $3.6 \mathrm{~s}$ had elapsed, pressing the spacebar again (Supp. Fig. 6). Following each duration production, we asked participants to assess their performance, which constitutes a metacognitive judgment task, and provide an assessment of temporal error monitoring (Akdoğan \& Balcı, 2017; Kononowicz et al., 2019). In temporal production tasks, the substantial variability within individuals that is observed is assumed to result from the endogenous timing uncertainty between trials. The statistical features of this timing variability and its relation to the time intervals being judged has been one of the primary focuses of the psychophysical study in interval timing (Grondin, 2001). The fact that organisms can access their level of endogenous timing uncertainty

as a form of temporal metacognition (Akdoğan \& Balcı, 2017; Kononowicz et al., 2019) might serve optimal temporal decisions in animals and humans (Balci, Freestone, et al., 2009). Hence, this novel metacognitive assessment of temporal judgments was included here by asking participants to not only evaluate the signed error magnitude of their temporal production (using a VAS) but also to rate their confidence either of their temporal production (Turkey) or of their metacognitive judgment (most countries). The descriptive statistics of the duration productions (s) for all participating countries in S1 are illustrated in Fig. $\mathbf{2 b}$.

\section{Spontaneous finger tapping (Free tapping)}

Spontaneous motor tempo, i.e., the rate at which an individual taps in the absence of any timing cue, is universally situated between around $1 \mathrm{~Hz}$ and $4 \mathrm{~Hz}$ with a bimodal or even trimodal distribution of the intertap intervals (peaks at around 250, 500 and 1000 ms; Hammerschmidt et al., 2021). The rate at which participants tap is assumed to reflect the speed or the period of a still largely unknown timekeeper, and it has been shown to be sensitive to alterations such as aging (McAuley et al., 2006; Turgeon \& Wing, 2012). This has been argued to be because spontaneous tapping tasks are too simple to be compensated by alternative compensatory mechanisms i.e., they are not cognitively penetrable (Turgeon et al., 2016). Basic information about the task is provided in (Supp. Fig. 7) and the descriptive statistics of the inter-tap-intervals (ITIS) for all participating countries in S1 are illustrated in Fig. 2a.

\section{Synchronization-Continuation}

In the field of motor timing, the classical synchronization-continuation paradigm (Semjen et al., 2000) consists of asking participants to synchronize their finger tapping with an auditory metronome and then to continue finger tapping with a sequence of constant intervals at the pace they initially synchronized with (Wing \& Kristofferson, 1973). In the continuation phase, the variability of the ITIs is the key dependent variable of interest (Wing \& Kristofferson, 1973). When the stimulus period is varied parametrically, an auto-correlation analysis of the series of produced intervals can be used to sort out the part of observed variability due to the temporal component of the task (associated to the underlying timing mechanism) and the one due to the implementation of the intervals with finger taps (the motor 
component). The synchronization phase, also known as paced finger tapping, is one of the simplest tasks to study sensorimotor synchronization, which has been argued to capture the ability of coordinating one's own movement with an external metronome (Repp \& Su, 2013). In paced finger tapping, it is the asynchrony (the time difference between response and stimulus) which is the fundamental variable of interest (Chen et al., 1997), both for isochronous and for perturbed sequences (Bavassi et al., 2013). A succinct illustration of the task used in the study is provided in Supp. Fig. 8. We tested two conditions: tapping in-sync or out-of-sync with the stimuli. The measured asynchronies in the synchronization task and the ITIs in the continuation task during lockdown are illustrated in Fig. 2a for all participating countries.

\section{Foreperiod paradigm and implicit timing}

The implicit extraction of temporal regularities from the environment allows forming temporal predictions and orienting attention to particular moments in time (Nobre \& Van Ede, 2018), which can lead to more efficient behavior, such as faster response times, or improved perceptual sensitivity (Cravo et al., 2011; Herbst \& Obleser, 2019). Here, we implemented an implicit timing task (Supp. Fig. 9), in which we varied the foreperiod (the time interval between a cue and target tone), such that the duration was either fixed (hence predictable) or variable (non-predictable) throughout a block, and measured response times as an index of efficient temporal prediction. The measured reaction times (RTs) in S1 for all participating countries are illustrated in Fig. 2c.

\section{Delay-Discounting}

Delay discounting refers to the devaluation of the reward amount as a function of delay to its receipt (Loewenstein, 1988), making the amount but also the proximity of the reward an important factor in determining the choice behavior of participants when they are asked to choose between two options. Some individuals may prefer the immediate reward over a delayed reward even when the amount offered immediately is substantially less than the amount offered after a delay (preferring to receive $\$ 5$ now over receiving $\$ 20$ in a year). Confinement is a condition that typically leads to the state of boredom, which can trigger impulsivity (Moynihan et al., 2017). In this task (Supp. Fig. 10), different amounts were offered to participants at different delays to estimate the subjective values of the offers as a function of time required to collect them.

\section{Self-preference}

Phenomenological approaches have related time with self as early as the 20th century (Husserl, 2012) leading psychiatrists to link time with disorders of the self in psychosis (Fuchs, 2007; Minkowski, 2013; Vogeley \& Kupke, 2007). Such a link has been evidenced experimentally (Foerster et al., 2021; Martin et al., 2017). In addition to the PQ-16 questionnaire exploring attenuated psychosis, the self-preference task was added to provide an objective self-related measure. It has repeatedly been shown that a stimulus we associate with ourselves is processed faster and with higher accuracy than a stimulus we associate with others (Makwana \& Srinivasan, 2019; Sui et al., 2012). These effects can either be accounted for by a 
self-referent memory advantage (Cunningham et al., 2008) or by enhanced attention drawn to selfrelated information (Keyes et al., 2010). To test this, and in accordance with previous work, we used a reaction time task in which participants learn to associate a geometrical shape with a label ("Self", "Friend" and "Other"; Supp. Fig. 11). On subsequent trials, participants are presented with one shape and one label, which may or not match with the previously learned associations. Participants had to report as fast and as accurately as possible whether the shape and the label matched.

\section{Accessing Data From The Blursday Database}

Our databasing approach follows the FAIR principles (Wilkinson et al., 2016) stipulating Findability, Accessibility, Interoperability, and Reusability of the data. In line with FAIR, we provide a graphical user interface to researchers, allowing them to easily and conveniently parse the data in a way that best fits their research needs (https://dnacombo.shinyapps.io/Blursday/). Data collected from each task and questionnaire are available at the individual trial/item level per participant in the database and will be incremented with quantified estimates per participant as analyses progress. The individual trial data are given to support modeling efforts that typically consider trial-based data sometimes based on the order of their occurrence. Due to the nature of the tasks, reliable timing is an important factor. To help researchers estimate the degree of timing uncertainty in data collected online (Bridges et al., 2020), we provide the participant's operating system and browser information with which data were collected. To improve the readability of the data at this level of presentation, we also provide human-readable readme.txt files for each questionnaire and task in a dedicated OSF repository (https://osf.io/359qm/ in folder Study_design/README/) along with additional sources of information that facilitates the reusability of the data.

\section{Data Reliability And Psychophysical Benchmarking Of Timing Measures}

In this section, we provide basic descriptive statistics for some of the collected data in Session 1 (S1) for several well-known timing tasks we included in the Blursday database (Figure 2).

In the spontaneous motor tapping task (Fig. 2a, left panel), the rate at which individuals spontaneously tapped approximated the expected inter-tap intervals (Hammerschmidt et al., 2021). Similarly, the asynchronies during the synchronization task showed narrow distributions around zero (Fig. 2a, middle panel), and the ITIs (Fig. 2a, right panel) during the continuation task showed a narrow distribution around the inter-stimulus interval (Repp, 2005; Repp \& Su, 2013; Semjen et al., 2000). In the temporal production task (Fig. 2b), participants showed a distribution of produced durations centered on a median of $3 \mathrm{~s}$ slightly below the instructed value of $3.6 \mathrm{~s}$ and with a variance expected for this range of suprasecond durations (Buhusi \& Oprisan, 2013; Kononowicz et al., 2019). In the auditory foreperiod paradigm (Fig. 2c), we see right-tailed distributions typical of reaction times (RTs) data with a mean/median of 177 $\mathrm{ms}$ and $162 \mathrm{~ms}$, respectively. These values are comparable to recently published work using a foreperiod paradigm in laboratory settings (Grabenhorst et al., 2019). As for the temporal landmarks task, the 
median value of their response time to the question "What day is it?" was $2.6 \mathrm{~s}$ also with right-tailed distributed (Fig. 2d) consistent with previous seminal work (Shanon, 1979).

\section{Sleep Disturbances (Psqi) And Anxiety And Depression Levels (Hads): A Cross-replication And Extension To The General Population In France}

Several independent studies using different types of questionnaires reported a high level of sleep disturbances during the first wave of the Covid-19 pandemic (e.g., Bertrand et al., 2021; Cellini et al., 2020; Trakada et al., 2020). During that same period, an increase of stress, anxiety, and depression was reported in the general population (Salari et al., 2020). In Blursday, we collected information about sleep difficulties using the PSQI (the most widely used self-report questionnaire to assess subjective sleep quality), as well as the level of anxiety and depression using the HADS. As the Blursday database provides the date of the data collection, comparisons across studies can be performed. We illustrate in Fig. 3 a cross-replication in which we compared the data collected in the French sample of Blursday between the $6^{\text {th }}$ and the $11^{\text {th }}$ of May 2020, with the data sampled by Bertrand and collaborators (2021) during the same period. Despite differences in sample size for that time period ( $\mathrm{N}=124$ in Blursday, $\mathrm{N}=$ 1627 in Bertrand et al., 2021) and in demographics (>18 y.o. in Blursday, <19 y.o. in Bertrand et al., 2021), the outcomes are remarkably similar.

\section{Assessment Of Objective And Subjective Confinement Indices}

The diversity of questionnaires included in the study provides a rich resource for numerous proxies of lockdown and subjective confinement indices. Herein, we illustrate four possible approaches illustrating the severity of lockdown experienced by an individual, objectively, semi-objectively, and subjectively.

A first objective measure (by country) is the Stringency Index derived by the OurWorldInData (Hale et al., 2021). The Stringency Index is a composite measure of nine governmental response indicators that include school closures, workplace closures, and travel bans, rescaled to a value ranging from 0 to 100 with 100 being the strictest stringency. A second objective measure (by country) can be found in the Google mobility index measures during Covid-19 (Google, 2021). For the purpose of our concise report, we selected the Transit Station Mobility Index (henceforth referred to as Mobility Index), which quantifies how much time visitors spent in various transit stations (subway, taxi stand, rentals) during a selected period relative to a baseline period. In the Google dataset, the baseline was defined as the median value from a five-week period spanning January $3^{\text {rd }}$ to February $6^{\text {th }}, 2020$. The more negative the mobility index, the less mobility compared to baseline. As visible in Fig. 1, while sessions were carefully aligned to the governmental lockdowns and state of emergency rules, they do not strictly map to the level of stringency or to the degree of mobility estimated in a given country. Some of our results and analyses indicate that objective measures of stringency and mobility may be adequate covariates to explore the effect of lockdown on behavior (for instance, see outcomes of our retrospective duration analysis below). 
A third measure of lockdown is the number of days participants reported being stranded at home in our confinement tracker. Although the verbal report of participants could be used as an objective measure, this estimation is prone to subjective factors that include memory (Huttenlocher et al., 1992), idiosyncratic and cultural biases (Koriat \& Fischhoff, 1974; Roediger \& Crowder, 1976) and variable temporal orientation towards socially meaningful events (Jonas \& Huguet, 2008). Hence, the reported number of days in lockdown cannot be considered a veridical and objective measure of lockdown, but it can minimally provide a fair and subjective approximation for it. The degree of deviance of the subjective measure with the veridical day of confinement could be compared to the locally applicable rules and official dates of lockdown for each participant although uncertainty as to each individual case remains. Hence, the reported number of days in lockdown is considered a semi-objective measure.

Fourth, we defined a measure of subjective confinement based on participants' self-assessed feeling of being isolated. Out of the 20 items which we tested using the full UCLA questionnaire (Russell, 1996; Russell et al., 1980), we used the ratings to only 5 items which could be directly related to the consequences of lockdown and stringency measures. We made a proxy for the feeling of confinement using these 5 selected items: "I have nobody to talk to", "I lack companionship", "I feel completely alone", "I feel starved for company", and "I feel isolated from others". Answers to these questions were obtained using a four-level Likert scale. While objective measures of confinement captured the situation well, a large inter-individual variability may subsist in participants' subjective feeling of having to stay home, being fully confined or under various stringency rules. This could be due to factors such as personality traits (which can be assessed with the BFI included in Blurdsay), or social isolation (which can be explored with the remaining items of UCLA and various questions such as changes in social media use, number of people in the household, and changes in habits included in Blursday). For this concise report, we chose a direct and minimal approach using a Confirmatory Factor Analysis (CFA), which showed that responses to the five items selected a priori, could be aggregated along two dimensions, which we refer to as "self-reported loneliness" (capturing responses to statements "I have...", "I lack...") and "felt loneliness" (capturing responses to "I feel..."). These dimensions are available in the database but for simplicity, we combined these two estimates of loneliness as a proxy for "subjective confinement", which scored anywhere between 5 (feeling very isolated) to 20 (not feeling isolated).

We integrated the objective lockdown states and subjective confinement measures in the Blursday database as an optional feature of data downloading. Below, we use these measures as covariates and illustrate their impact on subjective time in the sampled population.

\section{Temporal Distortions And Novel Empirical Observations}

We turn to novel quantitative observations regarding the effect of lockdowns on psychophysical measures of subjective temporality including retrospective duration estimation, and ratings of the felt passage of time and subjective temporal distances. 


\section{Retrospective duration on the scale of minutes to hours}

In the retrospective duration task, we asked participants to provide an estimate (in minutes, seconds) of how much time had elapsed since the last time they logged on to the study website, which was timestamped in the collected data. We used three criteria for defining outliers in our analysis: the first criterion rejected data points with a clock duration shorter than 1 minute and longer than 5 hours due to the implausibility of these timings in our study protocol. The second criterion excluded data with subjective retrospective durations shorter than $12 \mathrm{~s}$ and larger than 25 hours (i.e., subjective estimates that were 0.2 times the shortest possible clock duration and 5 times the longest possible clock duration). The last criterion removed data points with relative duration errors computed as [)] beyond the 95 central percentiles per country and per session. The application of these criteria discarded $8.8 \%$ of the original dataset.

\section{Retrospective duration estimates scale with clock duration}

Using a linear regression of the logarithm of retrospective duration estimates as a function of the logarithm of clock duration, separately for S1 (during lockdown) and for SC (outside of lockdown, control participants) showed that participants performed the task as expected in both sessions: retrospective duration estimates increased with increasing clock duration (Fig. 4A). Interestingly, the deviations of retrospective duration estimates from clock durations were scale-dependent such that all participants tended to overestimate the short durations and underestimate the long durations. This pattern is consistent with Vierordt's law and the central tendency effect typically reported in magnitude estimations (Glasauer \& Shi, 2021; Lejeune \& Wearden, 2009; Petzschner et al., 2015). The two regression lines had a slope below 1 (S1: 0.89 +/- 0.019 s.e.m.; S2: 0.77 +/- 0.042 s.e.m.).

Using a two way ANOVA with factors of Session (2: S1, SC) and clock duration, we confirmed a significant main effect of clock duration on subjective retrospective duration $\left(F(1,1739)=2462.05, p<2 \mathrm{e}^{-}\right.$ ${ }^{16}$ ) and a significant interaction between retrospective duration and session $(F(1,1739)=7.57, p=$ 0.0059). This interaction captures the observation that the regression slopes in each session differed significantly. To make this observation more tangible: a clock duration of 10 minutes was overestimated by $30 \mathrm{~s}+/-15 \mathrm{~s}$ during lockdown (S1) but by $92 \mathrm{~s}+/-36 \mathrm{~s}$ during the control session (SC). Conversely, a clock duration of 1 hour was underestimated by $7 \mathrm{~min}$ and $10 \mathrm{~s}+/-54$ seconds (thus, estimated as $\sim 52$ minutes) during lockdown (S1) but by 14 min and $20 \mathrm{~s}+/-108$ seconds (thus, estimated as $\sim 46$ minutes) outside of it (SC).

Intriguingly, our analyses revealed the existence of a possible "indifference interval" in retrospective duration estimation, which converge in both sessions at a relatively close clock duration of about a quarter of an hour: on average, participants estimated the duration most accurately (i.e., closest to clock duration) for clock durations of about 16 minutes during lockdown, and of about 18.5 minutes outside of it (SC). 
Next, as stringency and mobility indices did not strictly map with the experimental sessions (Fig. 1), we used data from all sessions (S1, S2, S3, S4, SC) and indexed the severity of lockdown using the stringency, the mobility, and the subjective confinement indices described in the previous section. We also included age and time of day as possible covariates. We modeled the error term related to participants as a random intercept using a linear mixed model approach on participants' relative retrospective duration estimates (as implemented in the R package Ime4; Bates et al., 2015). We quantified the relative duration estimations as retrospective duration estimation / clock duration to pull all temporal scales together. The standard deviation of the estimated random effects was smaller than that of the residuals, suggesting that random effects could be ignored and running a linear model with the same fixed effects retrieved similar outcomes; hence, we solely report the fixed effects analysis.

\section{Mobility affects retrospective duration estimates}

Using an ANOVA on the coefficient estimates revealed a highly significant effect of the stringency index $(F(1,2140)=8.54, p=0.003$; Fig. 4b) and of the mobility index $(F(1,2140)=8.12, p=0.004$; Fig. 4c $)$ on relative retrospective duration estimates. This effect indicated that an increase of 80 on the stringency scale (i.e., the entire range of available data) corresponded to a decrease of $30 \%$ in estimated retrospective duration so that the more stringent the governmental measures were, the shorter retrospective duration estimates were. Conversely, the mobility index suggested that the closer to normal mobility participants were, the shorter the retrospective duration were. Stringency and mobility distinctly affect retrospective duration. As seen in Fig. 4c, our sessions and data collections seemed to align well with the mobility index. We observed no significant effects of the subjective confinement index, age, or hour of day on retrospective duration estimates.

\section{Passage of time on the scale of a few days}

Next, we explored four subjective time measurements collected using participants' ratings on VAS: the passage of time (over a few days), the subjective temporal distance to the first day of lockdown (past temporal orientation) and the subjective temporal distances to one week and one month from now (future temporal orientation). For all four, we used a linear mixed effect model with covariates identical to those used for retrospective duration estimates, namely: stringency index, mobility index, subjective confinement index, age, and hour of day. For all measures of subjective temporal distances, a random intercept per participant was added.

To capture the temporal phenomenology of the felt passage of time, individuals can rate their feeling of how fast time is passing over a certain lapse of time using a VAS. This approach was used in previous studies (Droit-Volet et al., 2020, 2021; Martinelli et al., 2020; Ogden, 2020, 2021). In Blursday, we asked participants to rate their feeling of the passage of time over the last few days. With a linear regression approach, we found that the passage of time was significantly related to participant subjective 
confinement score $\left(F(1,1860)=28.44, p=1.087 \mathrm{e}^{-07} ;\right.$ Fig. 5$)$ so that the less isolated participants felt, the faster their ratings of the passage of time. Under the linear assumption (i.e., away from the boundaries of the VAS), the most extreme differences in subjective score of confinement (from 5 feeling most confined to 20 feeling least confined) corresponded, on average, to a difference of 13.8 on the passage of time VAS going from $53.9+/-1.7$ s.e.m to $67.7+/-1.3$ s.e.m.. No other tested factors were found to significantly affect the feeling of the passage of time in this analysis.

\section{Temporal Orientation and Subjective Temporal Distances to past (from the start of lockdown) and future (week and month scales)}

Participants reported on a VAS ranging from 0 (very close) to 100 (very far) their subjective temporal distance to the first day of lockdown (past orientation) or week and month to come (future orientation).

The subjective temporal distances to the first day of lockdown (past orientation) were significantly affected by the mobility $\left(F(1,3814)=80.25, p=2.2 \mathrm{e}^{-16}\right.$; Fig. $6 \mathrm{a}$, right panel) so that the closer to normalcy, the further away participants rated their first lockdown day to feel. Specifically, under the linear assumption (i.e. away from the VAS boundaries), the most extreme increase in mobility transit going from -90 to 0 corresponded, on average, to a difference of 23.5 points on the subjective temporal distance scale, which went from $52.9+/-1.2$ s.e.m to 76.4 +/- 1.8 s.e.m. Conversely, participants' subjective confinement scores significantly affected their subjective temporal distances to the first day of lockdown $(F(1,2742)=9.6, p=0.0019$; Fig. 6a, right panel). Under the linear assumption, the less subjectively isolated participant felt (going from 5 to 20 of subjective confinement score), the closer in time that day felt. On average, an increase of subjective confinement score (interpreted as feeling less isolated) of 7.3 yielded participants' subjective temporal distance to be rated as closer $(65.7+/-1.6$ s.e.m. to $58.4+/-1.1$ s.e.m.).

We then explored participants' (future) subjective temporal distances at two time scales, "next week" and "next month" (Fig. 6b, light and dark green, respectively). A first common effect to both time scales was driven by the age of participants (week: $F(1,910)=12.23, p=0.00049$; month: $F(1,943)=25.24, p=$ $6.04 \mathrm{e}^{-07}$; Fig. $6 \mathbf{c}$, top left and right panels, respectively). Under the linear assumption, the most extreme differences in age (going from 18 to 88 years old) corresponded to an average difference of -11.2 on the subjective temporal distance scale for "next week" (from $36.7+/-1.19$ s.e.m to $25.5+/-2.51$ s.e.m) and of -17.7 for "next month" (from $60.2+/-1.20$ s.e.m, to $42.5+/-2.74$ s.e.m). In other words, the older participants were, the shorter they rated their subjective distances to the future, whether week or month.

Participants' subjective confinement scores also significantly affected their subjective temporal distances at both time scales (week: $F(1,1437)=6.46, p=0.01$; month: $F(1,1486)=16.82, p=4.32 \mathrm{e}^{-05}$; Fig. 6c, middle left and right panels, respectively). The less isolated participants felt, the closer their subjective future felt. Under the linear assumption, the most extreme increase in subjective confinement score (from 
5 to 20) corresponded to an average difference of -7.09 on the subjective temporal distance scale for "next week" (from $31.0+$ +/- 1.34 s.e.m to 38.1 +/- 1.95 s.e.m.) and of -12.6 for "next month" (from $63.4+/$ 2.10 s.e.m to $50.7+/-1.38$ s.e.m).

Last, we observed an increase of felt distance to "next month" with an increase in stringency measures (month: $F(1,1163)=8.62, p=0.003$; Fig. 6c, bottom right panel). Under the linear assumption, the most extreme increase in stringency (from 25 to 90 ) corresponded to an average difference of 9.93 on participants' subjective temporal scale to "next month" (going from $49.5+/-2.28$ s.e.m, to $59.4+/-1.46$ s.e.m.)

\section{Discussion}

The Covid-19 pandemic is one of those rare historical episodes during which virtually the entire world adopted similar constraints on the human population at the same time. The initial measures against Covid-19 were state-of-emergencies and partial to full lockdowns. Herein, we report a comprehensive database, which provides tangible quantitative and qualitative assessments documenting a wide range of factors mediating the temporal distortions and disorientations experienced during this historical episode. Making accessible the database and the online tools to collect additional data is motivated by the observations that, as we write this paper, we have not yet returned to a global normalcy level. Our experimental tools have been and can be easily translated to additional languages, providing an easy stepping stone for inclusive cross-cultural studies using multidimensional variables. We made efforts for the Blursday database to be highly accessible and readable to all researchers. We provide a graphical user interface that researchers without programming background can use for parsing and retrieving the data as required by their research questions. We also include optional outcomes of some of our preliminary analyses as part of the data (e.g., objective lockdown measures and subjective confinement indices).

Herein, we wished to highlight the existence of Blursday as well as showcase the reliability of the collected data on measures like sleep disturbances and anxiety, and well-established empirical facts typically measured in laboratory settings. To this end, we provided a replication and extension of previously reported sleep disturbances and borderline anxiety during the first lockdown in France (Bertrand et al., 2021). This replication shows that questionnaires included in the database can be reliably exploited independently of research interests dedicated to timing research. A number of questionnaires in the database could be exploited on their own for epidemiology, demographic, psychiatry, or chronobiology, or as covariates of other experimental measurements provided in the database. We also illustrate the distributions of several dependent variables in some of the timing tasks measured in Blursday, all of which pointing to the psychophysical and psychometric utility of our experimental approach for future investigations dedicated to these timing properties.

Importantly, by exploring the effects of stringency, mobility, subjective confinement, age, and hour of day on several measures of subjective time (Table 2), we report novel empirical findings showing that both 
objective measure of lockdown and subjective measures of confinement influence participants' time perception at different time scales.

First, we found that participants' subjective confinement scores, designed to capture how isolated participants felt, systematically accounted for changes in temporal orientation at the scale of a few days to week and month. Notably, the more isolated participants felt, the slower time seemed to pass. This observation generalizes how feeling isolated contributes to temporal distortions and the felt slowing down of the passage of time, which has been noted in a Brazilian study (Cravo et al., 2021). It also accounts for the diversity of passage of time changes reported during lockdown and attributed to possible consequences of isolation such as boredom (Droit-Volet et al., 2020, 2021; Loose et al., 2021; Martinelli et al., 2020; Ogden, 2020, 2021). Thus, evaluating the degree to which participants felt isolated is an important factor that provides additional insights on participants' subjective well-being independently of the objective lockdown situation. Indeed, our analyses were applied to all sessions (in and out of lockdown) over months during which objective lockdown measures were difficult to track.

Table 2: Summary of findings. To illustrate the richness of Blursday, we selected only a few measurements testing participants' perception at different time scales (from minutes to month) and orientation (past/future). We explored the effect of stringency (objective index), mobility (objective index), subjective confinement (subjective isolation index), age and hour of day on distinct measures of subjective time. A green checkmark indicates a significant effect of the covariate on the time measurement; a red cross marks an absence of significant effect. This table provides a succinct summary of otherwise more complex effects subjected to the limitation of our selection of relevant factors and choice of statistical models. Size and directionality of the effects were described in the Results section.

\begin{tabular}{|c|c|c|c|c|c|c|c|}
\hline Time Scale & Orientation & Task & $\begin{array}{l}\text { Objective } \\
\text { Stringency }\end{array}$ & $\begin{array}{l}\text { Objective } \\
\text { Mobility }\end{array}$ & $\begin{array}{c}\text { Felt } \\
\text { Confinement } \\
\end{array}$ & Age & Time of day \\
\hline $\begin{array}{l}\text { Minutes to } \\
\text { Hours }\end{array}$ & Past & $\begin{array}{l}\text { Retrospective } \\
\text { duration }\end{array}$ & & & X & $X$ & $x$ \\
\hline Few days & Past & Passage of Time & $x$ & $X$ & & $X$ & $x$ \\
\hline $\begin{array}{l}\text { Few days to } \\
\text { weeks }\end{array}$ & Past & 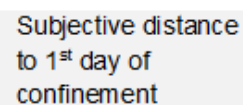 & $x$ & & & $X$ & $x$ \\
\hline Week & Future & $\begin{array}{l}\text { Subjective distance } \\
\text { to next week }\end{array}$ & $X$ & $X$ & & $v$ & $x$ \\
\hline Month & Future & $\begin{array}{l}\text { Subjective distance } \\
\text { to next month }\end{array}$ & & $x$ & & & $x$ \\
\hline
\end{tabular}

Interestingly, how isolated participants felt consistently modulated their subjective assessment of past and future distances so that the less isolated individuals felt, the closer in time past and future events seemed to be. These observations are essential in that temporal orientation is the ability to conceive of oneself in subjective (past or future) time and this ability has been argued to be a core component of autonoetic consciousness (Wheeler et al., 1997). We also found that the older the participants were, the 
closer their future appeared to be. Although previous timing research has shown differences on the perception of past and current passage of time (e.g. Winkler et al., 2017), future-oriented prospective timing has received less attention in the literature although it may entail differences in foresight across ages (Addis et al., 2008). Additional studies could use these measures together with participants' time perspective personality trait (ZTPI) to explore individuals' self-perception or the behavioral consequences of temporal disorientations on delay-discounting (all collected measures in the Blursday database).

Two objective indices of lockdown (stringency and mobility) affected retrospective duration estimations and with this task, we also observed a quite unexpected finding. It is now well-known that participants tested on a range of magnitudes display a behavior that conforms to the predictions of a Bayesian observer. Most studies assess this by using a range of magnitudes around a value of interest showing a generalizable pattern of central tendency or regression to the means (Glasauer \& Shi, 2021; Jazayeri \& Shadlen, 2010; Petzschner et al., 2015). The central tendency is described as a subjective underestimation of values below the mean of the distribution and an over-estimation of values above this mean. The range of magnitudes (e.g., the range of durations) calibrates the central value and, thus, the cutoff or the intersection between a presumed ideal observer (identity line) and an individual's subjective magnitude estimates. Our observed retrospective duration estimations conformed to this typical central tendency pattern otherwise known as Vierordt's law in timing research (Lejeune \& Wearden, 2009).

However, a key issue is that a classification as "short" or "long" does not have any reference point in our current study other than the prior belief of each unique participant. In Blursday, the range of retrospective durations explored was uniquely wide (from 1 minute to 5 hours) and was not controlled for since the duration was set by each participant's last login. Considering that each data sample was drawn from an independent observer tested on a different duration, the observation of a central tendency is unexpected and suggests the possibility of an indifference time interval of about a quarter of an hour.

Woodrow (1934) considered that the indifference interval should not be defined as the duration at which the individuals' average errors is zero but instead, as "the interval at which the average of all errors in any specified total distribution is zero". In other words, Woodrow posited the possibility of an absolute indifference interval. To demonstrate this, he tested participants using durations from 300 ms to 4000 ms and a temporal reproduction task; the temporal reproduction data showed a central tendency around $\sim 600 \mathrm{~ms}$. However, each participant was tested several times, which could not test the initial hypothesis. Closer to our retrospective duration task in which individual samples are independent, data in two reports showed a possible central tendency between 16-50 minutes (Yarmey, 2000) or between 2-3 minutes in another (Roy \& Christenfeld, 2008). In Blursday, the large-scale dataset, the wide-range of durations, and the multi-cultural inclusion make our observation quite unique: the observation of a central tendency towards a 15 to 20 minutes value at the inter-individual level is an intriguing and non-trivial novel observation. We can add to this that the central tendency for retrospective duration was significantly less pronounced during lockdown (S1) than outside of it (SC), so that the participants reports were closer to the ideal observer and conformed less to Vierordt's law during lockdown than outside of it. 
Finally, the highlighted observations by no means exhaustively exploit the rich repertoire of tasks, tests, and questionnaires of the Blursday database. This stands as a limitation of our results considering that we have not fully exploited the numerous factors that were recorded, and which could help further disentangle their weighted contribution to the temporal distortions we report. The Blursday database is amenable to cross-replication studies and currently feeds ongoing analyses with specific and narrower working hypotheses. For instance, with the innovative complementarity of questionnaires, a first crosscultural analysis revealed differences in anxiety and depression that can be partially attributed to differences in individuals and cultural time perspectives (Micillo et al., 2021). The motor timing tasks, designed to assess whether endogenous rhythms, sensorimotor productions and synchronizations were affected during isolation can be enriched with the personality traits collected on the same individuals as well as their demographics (age, gender). The foreperiod paradigm, which assesses implicit timing, is being explored with respect to some of the lockdown measures but also as a function of age, cultural diversity, and self-perception measures. The assessment of temporal landmarks taps into the possible distortions of temporal cognitive maps induced by the Covid-19 episode in the population, thereby enabling the exploration of how temporal distortions may link to episodic memory and participants' fluency reports.

\section{Conclusions}

In conclusion, we are confident that the Blursday dataset will act as a rich historical record of temporal disorientation and distortion during Covid-19 and serve as an empirical benchmark for future studies set out to use and build on the same tools to assess the effects of social isolation on temporal information processing across cultures and post-pandemic. We highlighted only a few possible observations drawn from the current dataset. Our study demonstrates the feasibility of a large and ambitious international study in a short time only thanks to the help of community builders such as the Timing Research Forum (TRF; http://timingforum.org). As members of the timing community, we hope that Blursday sets forth an international "TimeLab" that can support and foster multi-cultural large-scale studies in time and timing research.

\section{Code And Data Availability Project repository on OSF.io}

The OSF repository provides a public access platform to published materials, guides, and codes associated with the Blursday database (https://osf.io/359qm/). The OSF hub will be updated and incremented as results using the database get published. Readme files are provided describing the independent and dependent variables for each task, the number of trials, and the number of possible runs. We provide a comprehensive listing of translation and associated references for the validation of the questionnaires used in Blursday. Additional resources such as the dates of the sessions, the timelines, or useful resources for a more detailed assessment of local governmental measures are also provided. 


\section{Code repository Github.com}

https://github.com/dnacombo/TimeSocialDistancing

Server on Shinyapp.io

\section{Live: https://timesocialdistancing.shinyapps.io/Blursday/ and source code:_https://github.com/dnacombo/TSDshiny}

\section{Tasks and questionnaires Gorilla.sc}

All questionnaires and tasks used in the Blursday study are accessible as Open Materials in Gorilla in English (https://app.gorilla.sc/openmaterials/278377),

in French (https://app.gorilla.sc/openmaterials/27809),

in Greek (https://app.gorilla.sc/openmaterials/281196),

in Turkish (https://app.gorilla.sc/openmaterials/286114), and

in Japanese (https://app.gorilla.sc/openmaterials/286482).

\section{Declarations}

Competing interests: The authors declare no competing interests.

Licensing of the database : CC BY 4.0

Acknowledgments: We thank the many participants who took part in our extensive study, mostly without compensation and by sheer interest in citizen science. We are grateful to Bernadette Martins (CEA, NeuroSpin) for her continuous support on the ethical aspects of the protocol (CER-Paris-Saclay-2020020) and Maryline Hevin (CEA, NeuroSpin) for her administrative help. We thank numerous communication channels that have relayed and advertised the study: Christine Doublé (CEA, Neurospin), Laure Belot (Le Monde), and Cécile Chevallier (Le Parisien). We thank Dean Buonomano, Sylvie DroitVolet, Sonja Kotz, Natalia Martinelli, Ruth Ogden, Dan Poole, Darren Rhodes, and Hedderik van Rijn for their initial interest and support in building momentum to this international project. We thank Brill Publishing for sponsoring participation tokens in Gorilla. A.W. was funded by the doctoral school ED3C "Cerveau, Cognition, Comportement"; M.C. works in a core facility that receives funding from the program "Investissements d'avenir" (Agence Nationale de la Recherche, grant numbers ANR-10-IAIHU-06 and ANR11-INBS-006). S.G. was funded by the Natural Sciences and Engineering Research Council of Canada (NSERC); Y.Y. was funded by JSPS KAKENHI \#19H05308, UTokyo CiSHuB. V.vW. was funded by CEA and ANR-18-CE22-0016. No dedicated funding was received for this specific work. 
Conceptualization: V.vW, A.V., F.B., A.G.; Data curation: M.C., V.vW; Formal Analysis for this paper: M.C., V.vW, S.H., P.-A.R.; Funding acquisition: A.V., G.M., S.G., Y.Y., V.vW; Investigation: everyone; Methodology: everyone; Project administration: V.vW, A.V.; Resources: V.vW, A.V., S.G.; Software: everyone; Supervision: M.C., V.vW; Validation: everyone; Visualization: M.C., V.v.W, A.W., S.H., R.L., I.S., E.G.-H.; Writing - original draft: F.B., V.vW; Writing - review \& editing: everyone.

\section{References}

1. Addis, D. R., Wong, A. T., \& Schacter, D. L. (2008). Age-Related Changes in the Episodic Simulation of Future Events. Psychological Science, 19(1), 33-41. https://doi.org/10.1111/j.14679280.2008.02043.x

2. Akdoğan, B., \& Balcı, F. (2017). Are you early or late?: Temporal error monitoring. Journal of Experimental Psychology: General, 146(3), 347-361. https://doi.org/10.1037/xge0000265

3. Anwyl-Irvine, A. L., Massonnié, J., Flitton, A., Kirkham, N., \& Evershed, J. K. (2019). Gorilla in our midst: An online behavioral experiment builder. Behavior Research Methods. https://doi.org/10.3758/s13428-019-01237-x

4. Balci, F., Freestone, D., \& Gallistel, C. R. (2009). Risk assessment in man and mouse. Proceedings of the National Academy of Sciences, 106(7), 2459-2463.

5. Balci, F., Meck, W. H., Moore, H., \& Brunner, D. (2009). Timing deficits in aging and neuropathology. In Animal models of human cognitive aging (pp. 1-41). Springer.

6. Balcı, F., Freestone, D., Simen, P., Desouza, L., Cohen, J. D., \& Holmes, P. (2011). Optimal temporal risk assessment. Frontiers in Integrative Neuroscience, 5, 56.

7. Bates, D., Mächler, M., Bolker, B., \& Walker, S. (2015). Fitting Linear Mixed-Effects Models Using Ime4. Journal of Statistical Software, 067(i01). https://ideas.repec.org/a/jss/jstsof/v067i01.html

8. Bavassi, M. L., Tagliazucchi, E., \& Laje, R. (2013). Small perturbations in a finger-tapping task reveal inherent nonlinearities of the underlying error correction mechanism. Human Movement Science, 32(1), 21-47.

9. Bertrand, L., Schröder, C., Bourgin, P., Maruani, J., Atoui, Y., d'Ortho, M.-P., Lejoyeux, M., \& Geoffroy, P. A. (2021). Sleep and circadian rhythm characteristics in individuals from the general population during the French COVID-19 full lockdown. Journal of Sleep Research, e13480.

10. Block, R. A., Grondin, S., \& Zakay, D. (2018). Prospective and retrospective timing processes: Theories, methods, and findings. In Timing and time perception: Procedures, measures, \& applications (pp. 32-51). Brill.

11. Blume, C., Schmidt, M. H., \& Cajochen, C. (2020). Effects of the COVID-19 lockdown on human sleep and rest-activity rhythms. Current Biology.

12. Bridges, D., Pitiot, A., MacAskill, M. R., \& Peirce, J. W. (2020). The timing mega-study: Comparing a range of experiment generators, both lab-based and online. PeerJ, 8, e9414. 
13. Brown, S. W. (1997). Attentional resources in timing: Interference effects in concurrent temporal and nontemporal working memory tasks. Perception \& Psychophysics, 59(7), 1118-1140.

14. Buhusi, C. V., \& Oprisan, S. A. (2013). Time-scale invariance as an emergent property in a perceptron with realistic, noisy neurons. Behavioural Processes, 95, 60-70.

https://doi.org/10.1016/j.beproc.2013.02.015

15. Buonomano, D. V. (2007). The biology of time across different scales. Nature Chemical Biology, 3(10), 594.

16. Buysse, D. J., Reynolds III, C. F., Monk, T. H., Hoch, C. C., Yeager, A. L., \& Kupfer, D. J. (1991). Quantification of subjective sleep quality in healthy elderly men and women using the Pittsburgh Sleep Quality Index (PSQI). Sleep, 14(4), 331-338.

17. Cellini, N., Canale, N., Mioni, G., \& Costa, S. (2020). Changes in sleep pattern, sense of time and digital media use during COVID-19 lockdown in Italy. Journal of Sleep Research, e13074.

18. Chen, Y., Ding, M., \& Kelso, J. S. (1997). Long memory processes (1/f a type) in human coordination. Physical Review Letters, 79(22), 4501.

19. Cravo, A. M., de Azevedo, G. B., Azarias, C. M. B., Barne, L. C., Bueno, F. D., de Camargo, R. Y., Morita, V. C., Sirius, E., Recio, R. S., \& Silvestrin, M. (2021). Time experience in social isolation: A longitudinal study during the first months of COVID-19 pandemic in Brazil.

20. Cravo, A. M., Rohenkohl, G., Wyart, V., \& Nobre, A. C. (2011). Endogenous modulation of low frequency oscillations by temporal expectations. Journal of Neurophysiology, 106(6), 2964-2972.

21. Crawford, J. R., Henry, J. D., Crombie, C., \& Taylor, E. P. (2001). Normative data for the HADS from a large non-clinical sample. British Journal of Clinical Psychology, 40(4), 429-434.

22. Cunningham, S. J., Turk, D. J., Macdonald, L. M., \& Macrae, C. N. (2008). Yours or mine? Ownership and memory. Consciousness and Cognition, 17(1), 312-318.

23. D’Argembeau, A., Ortoleva, C., Jumentier, S., \& Van der Linden, M. (2010). Component processes underlying future thinking. Memory \& Cognition, 38(6), 809-819.

24. Dean, D. J., Tso, I. F., Giersch, A., Lee, H.-S., Baxter, T., Griffith, T., Song, L., \& Park, S. (2021). Crosscultural comparisons of psychosocial distress in the USA, South Korea, France, and Hong Kong during the initial phase of COVID-19. Psychiatry Research, 295, 113593.

25. Droit-Volet, S., Gil, S., Martinelli, N., Andant, N., Clinchamps, M., Parreira, L., Rouffiac, K., Dambrun, M., Huguet, P., \& Dubuis, B. (2020). Time and Covid-19 stress in the lockdown situation: Time free,«Dying» of boredom and sadness. PloS One, 15(8), e0236465.

26. Droit-Volet, S., Martinelli, N., Chevalère, J., Belletier, C., Dezecache, G., Gil, S., \& Huguet, P. (2021). The persistence of slowed time experience during the COVID-19 pandemic: Two longitudinal studies in France. Frontiers in Psychology, 12.

27. Fiorenzato, E., Zabberoni, S., Costa, A., \& Cona, G. (2021). Cognitive and mental health changes and their vulnerability factors related to COVID-19 lockdown in Italy. PLOS ONE, 16(1), e0246204. https://doi.org/10.1371/journal.pone.0246204 
28. Foerster, F. R., Weibel, S., Poncelet, P., Dufour, A., Delevoye-Turrell, Y., Capobianco, A., Ott, L., \& Giersch, A. (2021). Volatility of subliminal haptic feedbacks alters the feeling of control in schizophrenia.

29. Fortin, C., \& Breton, R. (1995). Temporal interval production and processing in working memory. Perception \& Psychophysics, 57(2), 203-215.

30. Friedman, W. J. (1993). Memory for the time of past events. Psychological Bulletin, 113(1), 44.

31. Fuchs, T. (2007). The temporal structure of intentionality and its disturbance in schizophrenia. Psychopathology, 40(4), 229-235. https://doi.org/10.1159/000101365

32. Ghotbi, N., Pilz, L. K., Winnebeck, E. C., Vetter, C., Zerbini, G., Lenssen, D., Frighetto, G., Salamanca, M., Costa, R., \& Montagnese, S. (2020). The $\mu$ MCTQ: An ultra-short version of the Munich ChronoType questionnaire. Journal of Biological Rhythms, 35(1), 98-110.

33. Glasauer, S., \& Shi, Z. (2021). The origin of Vierordt's law: The experimental protocol matters. PsyCh Journal.

34. Google, L. (n.d.). Google COVID-19 Community Mobility Reports. Retrieved October 4, 2021, from https://www.google.com/covid19/mobility/

35. Grabenhorst, M., Michalareas, G., Maloney, L. T., \& Poeppel, D. (2019). The anticipation of events in time. Nature Communications, 10(1), 5802. https://doi.org/10.1038/s41467-019-13849-0

36. Grondin, S. (2001). From physical time to the first and second moments of psychological time. Psychological Bulletin, 127(1), 22.

37. Grondin, S., \& Macar, F. (1992). Dividing attention between temporal and nontemporal tasks: A performance operating characteristic-POC-analysis. In Time, action and cognition (pp. 119-128). Springer.

38. Grondin, S., \& Plourde, M. (2007). Judging multi-minute intervals retrospectively. The Quarterly Journal of Experimental Psychology, 60(9), 1303-1312.

39. Hale, T., Angrist, N., Goldszmidt, R., Kira, B., Petherick, A., Phillips, T., Webster, S., Cameron-Blake, E., Hallas, L., Majumdar, S., \& Tatlow, H. (2021). A global panel database of pandemic policies (Oxford COVID-19 Government Response Tracker). Nature Human Behaviour, 5(4), 529-538.

https://doi.org/10.1038/s41562-021-01079-8

40. Hammerschmidt, D., Frieler, K., \& Wöllner, C. (2021). Spontaneous motor tempo: Investigating psychological, chronobiological, and demographic factors in a large-scale online tapping experiment. Frontiers in Psychology, 12, 2338.

41. Henry, J. D., \& Crawford, J. R. (2004). A meta-analytic review of verbal fluency performance in patients with traumatic brain injury. Neuropsychology, 18(4), 621.

42. Herbst, S. K., \& Obleser, J. (2019). Implicit temporal predictability enhances pitch discrimination sensitivity and biases the phase of delta oscillations in auditory cortex. Neurolmage, 203, 116198. https://doi.org/10.1016/j.neuroimage.2019.116198

43. Hicks, R. E. (1992). Prospective and retrospective judgments of time: A neurobehavioral analysis. In Time, action and cognition (pp. 97-108). Springer. 
44. Holman, E. A., \& Grisham, E. L. (2020). When time falls apart: The public health implications of distorted time perception in the age of COVID-19. Psychological Trauma: Theory, Research, Practice, and Policy, 12(S1), S63. https://doi.org/10.1037/tra0000756

45. Husserl, E. (2012). On the phenomenology of the consciousness of internal time (1893-1917) (Vol. 4). Springer Science \& Business Media.

46. Huttenlocher, J., Hedges, L. V., \& Prohaska, V. (1992). Memory for day of the week: A 5+ 2 day cycle. Journal of Experimental Psychology: General, 121(3), 313.

47. Ising, H. K., Veling, W., Loewy, R. L., Rietveld, M. W., Rietdijk, J., Dragt, S., Klaassen, R. M., Nieman, D. H., Wunderink, L., \& Linszen, D. H. (2012). The validity of the 16-item version of the Prodromal Questionnaire (PQ-16) to screen for ultra high risk of developing psychosis in the general helpseeking population. Schizophrenia Bulletin, 38(6), 1288-1296.

48. Jazayeri, M., \& Shadlen, M. N. (2010). Temporal context calibrates interval timing. Nature Neuroscience, 13(8), 1020-1026. https://doi.org/10.1038/nn.2590

49. Jonas, K. J., \& Huguet, P. (2008). What day is today? A social-psychological investigation into the process of time orientation. Personality and Social Psychology Bulletin, 34(3), 353-365.

50. Keyes, H., Brady, N., Reilly, R. B., \& Foxe, J. J. (2010). My face or yours? Event-related potential correlates of self-face processing. Brain and Cognition, 72(2), 244-254.

51. Kononowicz, T. W., Roger, C., \& van Wassenhove, V. (2019). Temporal metacognition as the decoding of self-generated brain dynamics. Cerebral Cortex, 29(10), 4366-4380.

52. Koriat, A., \& Fischhoff, B. (1974). What day is today? An inquiry into the process of time orientation. Memory \& Cognition, 2(2), 201-205.

53. Koriat, A., Fischhoff, B., \& Razel, O. (1976). An inquiry into the process of temporal orientation. Acta Psychologica, 40(1), 57-73.

54. Kraft, J. D., Grant, D. M., Taylor, D. L., Frosio, K. E., Nagel, K. M., \& Deros, D. E. (2019). Assessing the psychometric properties of the Attentional Style Questionnaire. Cognition and Emotion.

55. Laje, R., Agostino, P. V., \& Golombek, D. A. (2018). The times of our lives: Interaction among different biological periodicities. Frontiers in Integrative Neuroscience, 12, 10.

56. Lejeune, H., \& Wearden, J. H. (2009). Vierordt's The Experimental Study of the Time Sense (1868) and its legacy. European Journal of Cognitive Psychology, 21(6), 941-960.

57. Liberman, N., \& Trope, Y. (2014). Traversing psychological distance. Trends in Cognitive Sciences, 18(7), 364-369. https://doi.org/10.1016/j.tics.2014.03.001

58. Lieberoth, A., Lin, S.-Y., Stöckli, S., Han, H., Kowal, M., Gelpi, R., Chrona, S., Tran, T. P., Jeftić, A., Rasmussen, J., Cakal, H., Milfont, T. L., \& null, null. (2021). Stress and worry in the 2020 coronavirus pandemic: Relationships to trust and compliance with preventive measures across 48 countries in the COVIDiSTRESS global survey. Royal Society Open Science, 8(2), 200589. https://doi.org/10.1098/rsos.200589 
59. Loewenstein, G. F. (1988). Frames of mind in intertemporal choice. Management Science, 34(2), 200-214.

60. Loose, T., Wittmann, M., \& Vásquez-Echeverría, A. (2021). Disrupting times in the wake of the pandemic: Dispositional time attitudes, time perception and temporal focus. Time \& Society, $0961463 \times 211027420$.

61. Macar, F., Grondin, S., \& Casini, L. (1994). Controlled attention sharing influences time estimation. Memory \& Cognition, 22(6), 673-686.

62. Makwana, M., \& Srinivasan, N. (2019). Self-associated stimuli produce stronger intentional binding. Journal of Experimental Psychology: Human Perception and Performance, 45(11), 1436.

63. Marelli, S., Castelnuovo, A., Somma, A., Castronovo, V., Mombelli, S., Bottoni, D., Leitner, C., Fossati, A., \& Ferini-Strambi, L. (2021). Impact of COVID-19 lockdown on sleep quality in university students and administration staff. Journal of Neurology, 268(1), 8-15. https://doi.org/10.1007/s00415-02010056-6

64. Martin, B., Franck, N., Cermolacce, M., Falco, A., Benair, A., Etienne, E., Weibel, S., Coull, J. T., \& Giersch, A. (2017). Fragile temporal prediction in patients with schizophrenia is related to minimal self disorders. Scientific Reports, 7(1), 1-10.

65. Martinelli, N., Gil, S., Belletier, C., Chevalère, J., Dezecache, G., Huguet, P., \& Droit-Volet, S. (2020). Time and Emotion in the lockdown for the Covid-19 epidemic: The determinants of our experience of time? Frontiers in Psychology, 11, 3738.

66. McAuley, J. D., Jones, M. R., Holub, S., Johnston, H. M., \& Miller, N. S. (2006). The time of our lives: Life span development of timing and event tracking. Journal of Experimental Psychology: General, 135(3), 348.

67. Micillo, L., Rioux, P.-A., Mendoza, E., Kübel, S., Cellini, N., Wassenhove, V. van, Grondin, S., \& Mioni, G. (2021). Time Perspective predicts levels of Anxiety and Depression during the COVID-19 outbreak: A cross-cultural study. PsyArXiv. https://doi.org/10.31234/osf.io/8tqap

68. Minkowski, E. (2013). Le temps vécu. Presses Universitaires de France. https://doi.org/10.3917/puf.mink.2013.01

69. Nobre, A. C., \& Van Ede, F. (2018). Anticipated moments: Temporal structure in attention. Nature Reviews Neuroscience, 19(1), 34.

70. Ogden, R. (2020). The passage of time during the UK Covid-19 lockdown. Plos One, 15(7), e0235871.

71. Ogden, R. (2021). Distortions to the passage of time during England's second national lockdown: A role for depression. Plos One, 16(4), e0250412.

72. Petzschner, F. H., Glasauer, S., \& Stephan, K. E. (2015). A Bayesian perspective on magnitude estimation. Trends in Cognitive Sciences, 19(5), 285-293.

73. Polti, I., Martin, B., \& van Wassenhove, V. (2018). The effect of attention and working memory on the estimation of elapsed time. Scientific Reports, 8(1). https://doi.org/10.1038/s41598-018-25119-y 
74. Rammstedt, B. (2007). The 10-item Big Five Inventory: Norm values and investigation of sociodemographic effects based on a German population representative sample. European Journal of Psychological Assessment, 23(3), 193.

75. Rammstedt, B., \& John, O. P. (2007). Measuring personality in one minute or less: A 10-item short version of the Big Five Inventory in English and German. Journal of Research in Personality, 41(1), 203-212.

76. Randler, C. (2013). German version of the reduced Morningness-Eveningness Questionnaire (rMEQ). Biological Rhythm Research, 44(5), 730-736.

77. Repp, B. H. (2005). Sensorimotor synchronization: A review of the tapping literature. Psychonomic Bulletin \& Review, 12(6), 969-992. https://doi.org/10.3758/BF03206433

78. Repp, B. H., \& Su, Y.-H. (2013). Sensorimotor synchronization: A review of recent research (20062012). Psychonomic Bulletin \& Review, 20(3), 403-452.

79. Ritchie, H., Mathieu, E., Rodés-Guirao, L., Appel, C., Giattino, C., Ortiz-Ospina, E., Hasell, J., Macdonald, B., Beltekian, D., \& Roser, M. (2020). Coronavirus Pandemic (COVID-19). Our World in Data. https://ourworldindata.org/coronavirus-source-data

80. Roediger, H. L., \& Crowder, R. G. (1976). A serial position effect in recall of United States presidents. Bulletin of the Psychonomic Society, 8(4), 275-278.

81. Ross, M., \& Wilson, A. E. (2002). It feels like yesterday: Self-esteem, valence of personal past experiences, and judgments of subjective distance. Journal of Personality and Social Psychology, 82(5), 792.

82. Roy, M. M., \& Christenfeld, N. J. S. (2008). Effect of task length on remembered and predicted duration. Psychonomic Bulletin \& Review, 15(1), 202-207. https://doi.org/10.3758/PBR.15.1.202

83. Russell, D. W. (1996). UCLA Loneliness Scale (Version 3): Reliability, validity, and factor structure. Journal of Personality Assessment, 66(1), 20-40.

84. Russell, D. W., Peplau, L. A., \& Cutrona, C. E. (1980). The revised UCLA Loneliness Scale: Concurrent and discriminant validity evidence. Journal of Personality and Social Psychology, 39(3), 472.

85. Salari, N., Hosseinian-Far, A., Jalali, R., Vaisi-Raygani, A., Rasoulpoor, S., Mohammadi, M., Rasoulpoor, S., \& Khaledi-Paveh, B. (2020). Prevalence of stress, anxiety, depression among the general population during the COVID-19 pandemic: A systematic review and meta-analysis. Globalization and Health, 16(1), 1-11.

86. Schmidt, C., Collette, F., Cajochen, C., \& Peigneux, P. (2007). A time to think: Circadian rhythms in human cognition. Cognitive Neuropsychology, 24(7), 755-789.

87. Semjen, A., Schulze, H.-H., \& Vorberg, D. (2000). Timing precision in continuation and synchronization tapping. Psychological Research, 63(2), 137-147.

88. Shanon, B. (1979). Yesterday, today and tomorrow. Acta Psychologica, 43(6), 469-476.

89. Sircova, A., Van De Vijver, F. J., Osin, E., Milfont, T. L., Fieulaine, N., Kislali-Erginbilgic, A., Zimbardo, P. G., country), I. T. P. R. P. (presented in alphabetical order by, Djarallah, S., \& Chorfi, M. S. (2014). A 
global look at time: A 24-country study of the equivalence of the Zimbardo Time Perspective Inventory. Sage Open, 4(1), 2158244013515686.

90. Smyth, C. (1999). The Pittsburgh sleep quality index (PSQI). SLACK Incorporated Thorofare, NJ.

91. Sui, J., He, X., \& Humphreys, G. W. (2012). Perceptual effects of social salience: Evidence from selfprioritization effects on perceptual matching. Journal of Experimental Psychology: Human Perception and Performance, 38(5), 1105.

92. Thönes, S., \& Stocker, K. (2019). A standard conceptual framework for the study of subjective time. Consciousness and Cognition, 71, 114-122.

93. Trakada, A., Nikolaidis, P. T., Andrade, M. dos S., Puccinelli, P. J., Economou, N.-T., Steiropoulos, P., Knechtle, B., \& Trakada, G. (2020). Sleep during "lockdown" in the COVID-19 pandemic. International Journal of Environmental Research and Public Health, 17(23), 9094.

94. Turgeon, M., Lustig, C., \& Meck, W. H. (2016). Cognitive aging and time perception: Roles of bayesian optimization and degeneracy. Frontiers in Aging Neuroscience, 8, 102.

95. Turgeon, M., \& Wing, A. M. (2012). Late onset of age-related difference in unpaced tapping with no age-related difference in phase-shift error detection and correction. Psychology and Aging, 27(4), 1152.

96. Van Calster, L., D’Argembeau, A., \& Majerus, S. (2018). Measuring individual differences in internal versus external attention: The attentional style questionnaire. Personality and Individual Differences, $128,25-32$.

97. Vogeley, K., \& Kupke, C. (2007). Disturbances of Time Consciousness From a Phenomenological and a Neuroscientific Perspective. Schizophrenia Bulletin, 33(1), 157-165.

https://doi.org/10.1093/schbul/sbl056

98. Walach, H., Buchheld, N., Buttenmüller, V., Kleinknecht, N., \& Schmidt, S. (2006). Measuring mindfulness-The Freiburg mindfulness inventory (FMI). Personality and Individual Differences, $40(8), 1543-1555$.

99. Wearden, J. H. (2015). Passage of time judgements. Consciousness and Cognition, 38, 165-171. https://doi.org/10.1016/j.concog.2015.06.005

100. Wheeler, M. A., Stuss, D. T., \& Tulving, E. (1997). Toward a theory of episodic memory: The frontal lobes and autonoetic consciousness. Psychological Bulletin, 121(3), 331-354.

https://doi.org/10.1037/0033-2909.121.3.331

101. Wilkinson, M. D., Dumontier, M., Aalbersberg, Ij. J., Appleton, G., Axton, M., Baak, A., Blomberg, N., Boiten, J.-W., da Silva Santos, L. B., Bourne, P. E., Bouwman, J., Brookes, A. J., Clark, T., Crosas, M., Dillo, I., Dumon, O., Edmunds, S., Evelo, C. T., Finkers, R., ... Mons, B. (2016). The FAIR Guiding Principles for scientific data management and stewardship. Scientific Data, 3(1), 160018. https://doi.org/10.1038/sdata.2016.18

102. Wing, A. M., \& Kristofferson, A. B. (1973). Response delays and the timing of discrete motor responses. Perception \& Psychophysics, 14(1), 5-12. 
103. Winkler, I., Fischer, K., Kliesow, K., Rudolph, T., Thiel, C., \& Sedlmeier, P. (2017). Has it really Been that Long? Why Time Seems to Speed up with Age. Timing \& Time Perception, 5(2), 168-189. https://doi.org/10.1163/22134468-00002088

104. Wittmann, M., \& Paulus, M. P. (2008). Decision making, impulsivity and time perception. Trends in Cognitive Sciences, 12(1), 7-12.

105. Woodrow, H. (1934). The temporal indifference interval determined by the method of mean error. Journal of Experimental Psychology, 17(2), 167.

106. Yamada, Y., Ćepulić, D.-B., Coll-Martín, T., Debove, S., Gautreau, G., Han, H., Rasmussen, J., Tran, T. P., Travaglino, G. A., \& Lieberoth, A. (2021). COVIDiSTRESS Global Survey dataset on psychological and behavioural consequences of the COVID-19 outbreak. Scientific Data, 8(1), 1-23.

107. Yarmey, A. D. (2000). Retrospective duration estimations for variant and invariant events in field situations. Applied Cognitive Psychology, 14(1), 45-57. https://doi.org/10.1002/(SICI)10990720(200001)14:1<45::AID-ACP623>3.0.C0;2-U

108. Zakay, D., \& Block, R. A. (1997). Temporal cognition. Curr Dir Psychol Sci, 6(1), 12-16.

109. Zimbardo, P. G. (1990). The Stanford time perspective inventory. Stanford, CA: Stanford University.

110. Zimbardo, P. G., \& Boyd, J. N. (2015). Putting time in perspective: A valid, reliable individualdifferences metric. In Time perspective theory; review, research and application (pp. 17-55). Springer.

\section{Figures}

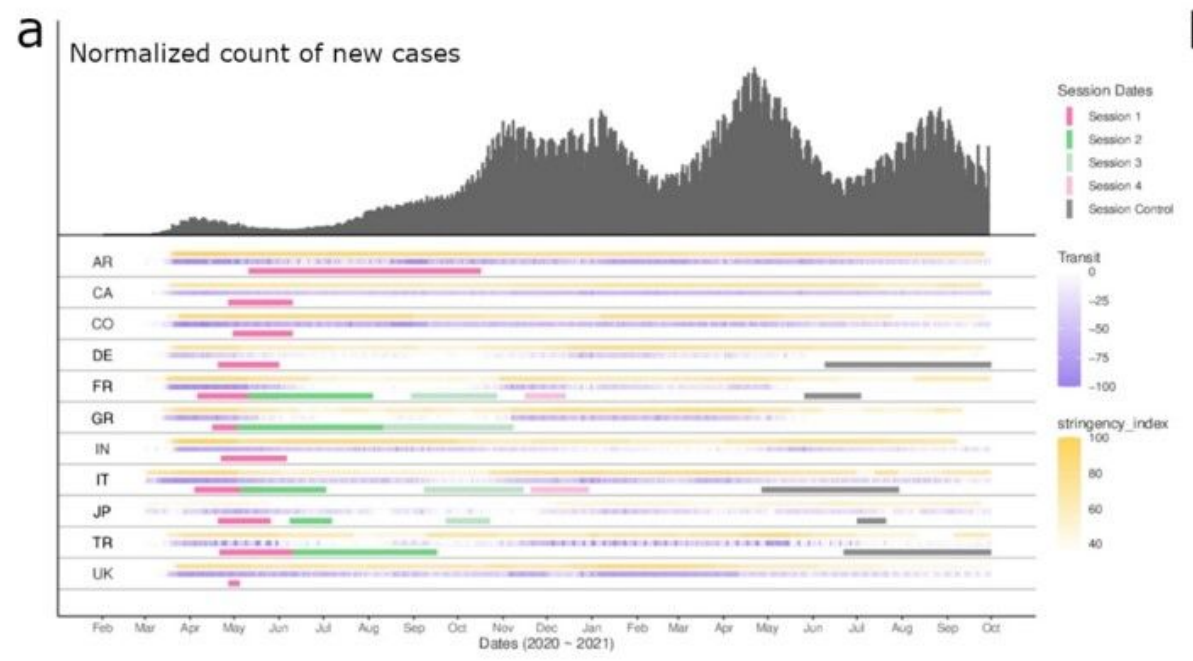

b

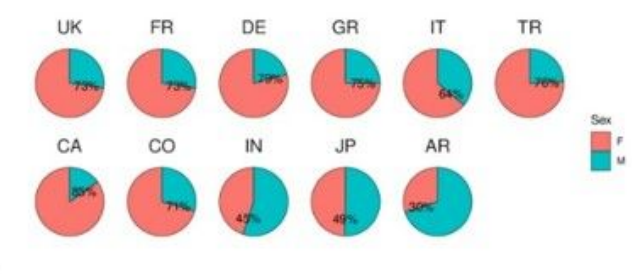

C

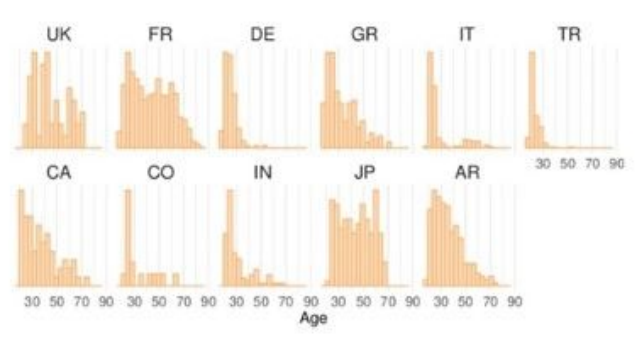

\section{Figure 1}

Data collection schedule, stringency index, mobility index and general demographics. Panel a: Sessions 1 and 4 (S1 and S4, respectively) took place during the first and the second lockdown or state-of-emergency of each country. Sessions 2 and 3 (S2 and S3, respectively) were set at least 2 weeks and 3 months away from the first lockdown in each country. The control session (SC) tested (and continues testing) a group 
of naive participants on the same set of questionnaires and tasks as those tested in S1. For illustration purposes, the daily Covid-19 cases (WHO COVID-19 Dashboard. Geneva: World Health Organization, 2020., 2021) were normalized by dividing the counts of daily Covid-19 cases by the total number of cases per country. Note that the number of cases in the initial start of the pandemic was likely underestimated. The stringency (Hale et al., 2021; Ritchie et al., 2020) and mobility indices (Google, 2021) were used as objective indicators of the state of each country at the time of data collection and are described in more detail in the "Assessment of objective and subjective confinement indices" section. Due to the varied timelines of emergency, stay-at-home or lockdown measures, post-hoc indices provide reliable and richer measures than ad-hoc sessioning. We illustrate this point in the Results section. Panel b: Proportion of males and females per country across all sessions. Panel c: Age distribution per country across all sessions. UK: United Kingdom; FR: France; DE: Germany; GR: Greece; IT: Italy; TR: Turkey; CA: Canada; CO: Colombia; IN: India; JP: Japan; AR: Argentina. Note that all figures were designed accounting for color blindness (Nichols, 2021). 

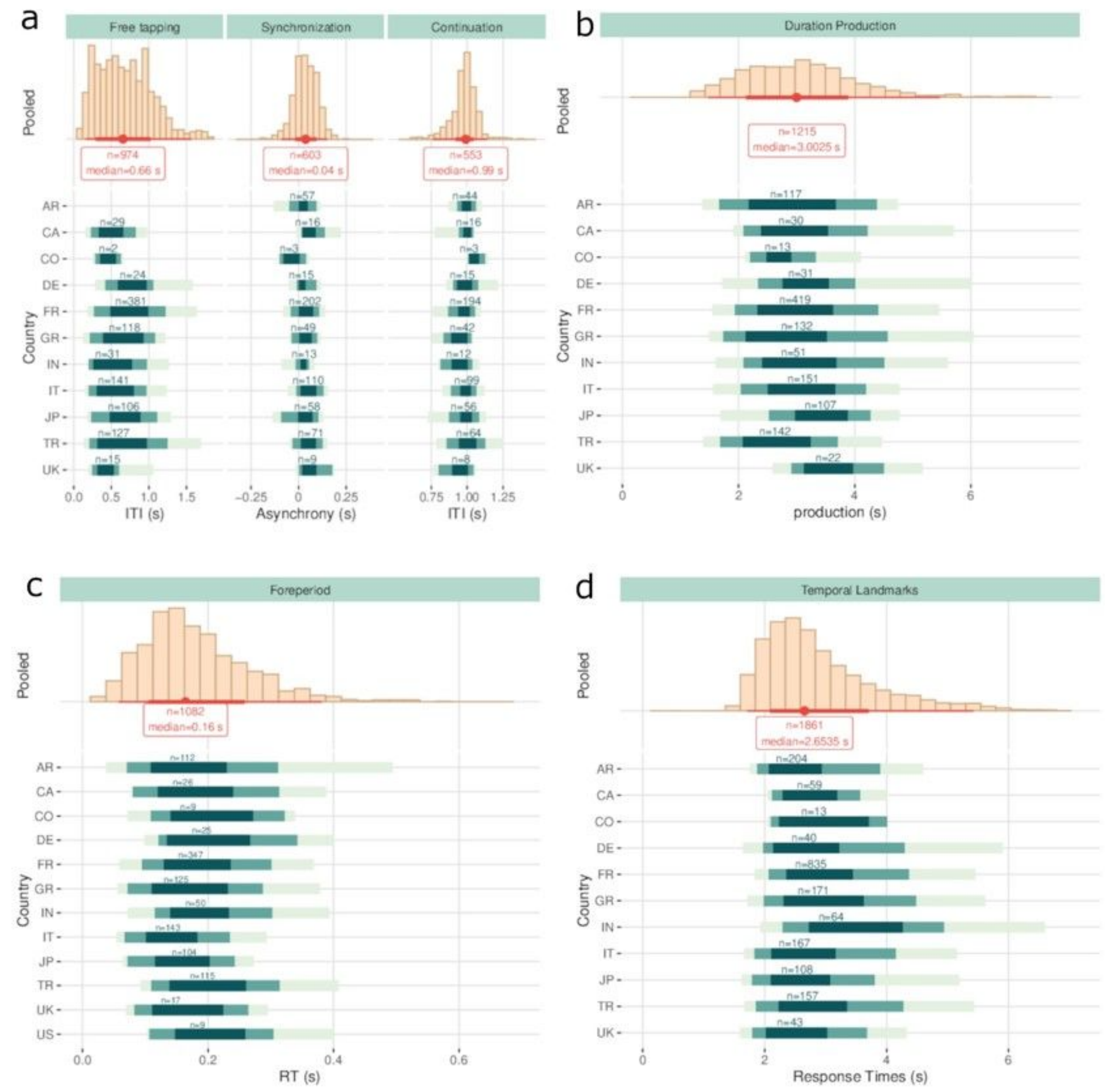

Figure 2

Sample descriptive statistics during lockdown (S1). The distributions of the main dependent variables in $\mathrm{S} 1$ are shown for a selection of tasks, pulled across all samples of all countries (top, orange). The thin line, thick line, and dot below each distribution show the $0.95,0.8$, and 0.5 (i.e. median) two-sided distribution quantiles, respectively. Similarly, the shaded lines below the histogram show the breakdown of this information or per country (bottom, green). Panel a: In the free tapping or spontaneous motor tapping task (left panel), we report the inter-tap-interval (ITI) computed as the difference between the occurrence times of consecutive taps. In the synchronization-continuation task, we computed the 
asynchrony as the time difference between each response and its corresponding visual stimulus in the synchronization phase (middle panel) and again the ITI in the continuation part of the task (right panel). Panel b: Drawn from the temporal duration production and metacognition task, we report the distribution of duration productions to the target $3.6 \mathrm{~s}$. Panel c: In the auditory foreperiod paradigm assessing implicit timing, we report reaction times (RTs) elicited by the presentation of the auditory target for all conditions. RTs exceeding the mean by \pm 2.5 standard deviations (per participant) were discarded as outliers. Panel $\mathrm{d}$ : In the temporal landmarks, we report the distribution of correct response times to the question "What day is it?"; response times longer than $8 \mathrm{~s}$ and beyond the $95 \%$ central percentiles were disregarded. All timings are provided in seconds (s). AR: Argentina; CA: Canada; CO: Colombia; DE: Germany; FR: France; GR: Greece; IN: India; IT: Italy; JP: Japan; TR: Turkey; UK: United Kingdom; US: United States of America.

\section{French data collected in the last week of the first lockdown (S1) May 6-11, 2020}

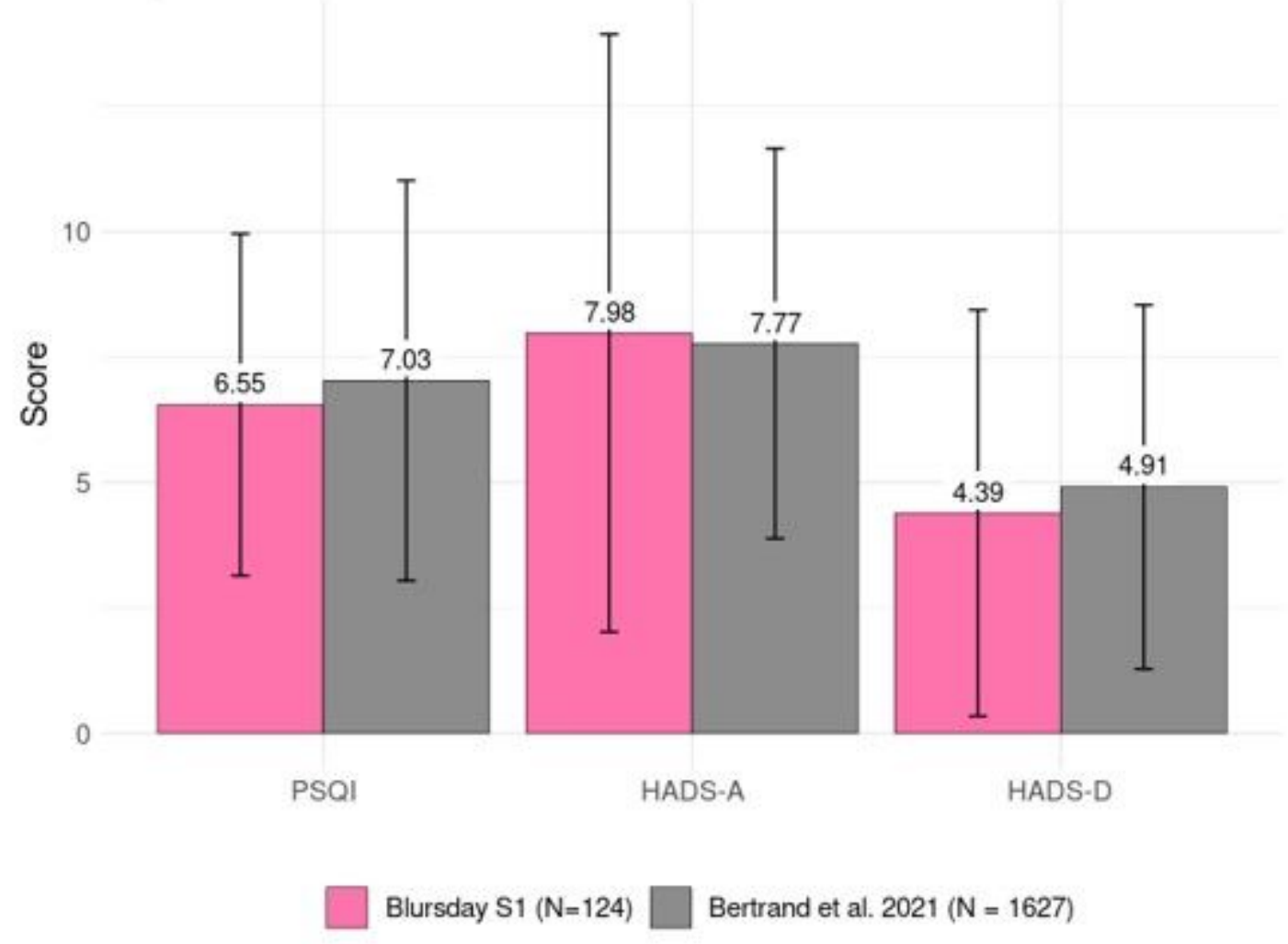

\section{Figure 3}

Comparison of PSQI and HADS scores between Blursday and Bertrand and collaborators (2021). We sampled the Blusday database (green) to match the country (France) and the collection period of Bertrand et al. (2021; gray) from May 6th to 11th 2020. PSQI: Pittsburgh Sleep Quality Index. The maximal scoring for PSQI is 21: the observed means of $\sim 7$ in both studies suggest that on average, participants reported poor sleep (cut-off $\geq 5$ ). HADS-A: Hospital Anxiety and Depression Scale - Anxiety Scale; HADS-D: Hospital Anxiety and Depression Scale - Depression Scale. The maximum scores for 
HADS-A and HADS-D are 21. The score of $~ 8$ in HADS-A indicates that, on average, participants reported a borderline level of anxiety (cut-off $\geq 8$ ) with a normal level of depression symptomatology scoring $\sim 5$ (cut-off $<8$ ). Error bars represent \pm S.D.

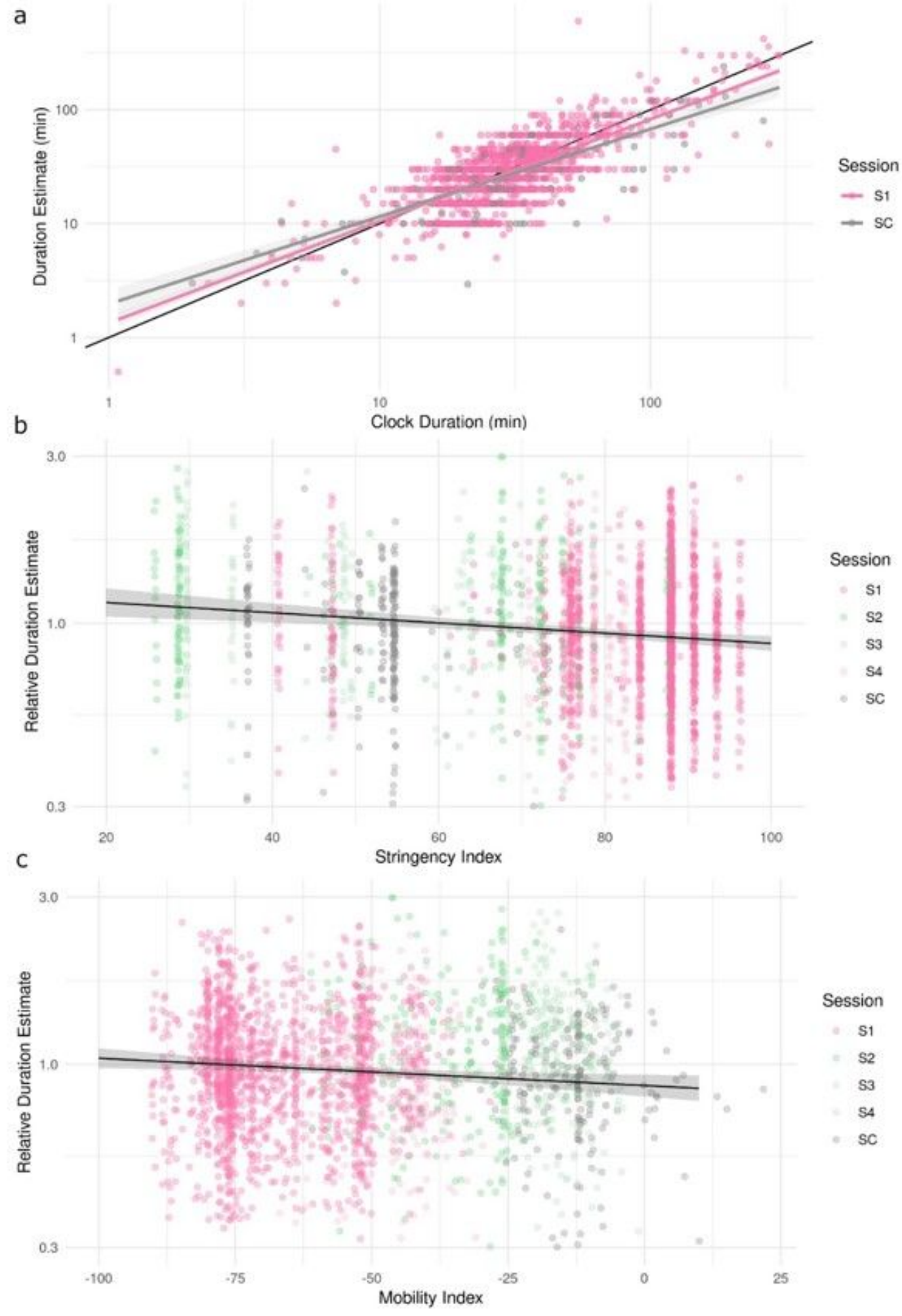

Figure 4

Retrospective duration estimation is affected by lockdown, stringency and mobility index. Panel a: Retrospective duration estimates (minutes) as a function of veridical clock duration (minutes) during 
lockdown (S1; pink) and outside of it (SC; gray). Each dot is a single participant. Regression lines were estimated from the linear mixed effect model with their $95 \%$ confidence interval in gray shading. Panel b: Relative retrospective duration estimates (unitless) as a function of the stringency index (a.u. between 0 and 100) for all sessions (colored). Colored dots are individual data points per participant and per session. Regression lines were estimated from the linear model with the 95\% confidence interval (gray shade). The more stringent governmental rules were, the shorter retrospective durations were estimated to be. Panel c: Relative retrospective duration estimates (no unit.) as a function of the mobility index (percent change relative to baseline, prior to lockdown, see main text) for all sessions (colored). Each dot is an individual data point per participant and per session. Black lines are regression lines estimated from the linear model with the $95 \%$ confidence interval reported in gray shading. The closer to baseline mobility, the shorter retrospective durations were estimated to be. 
a
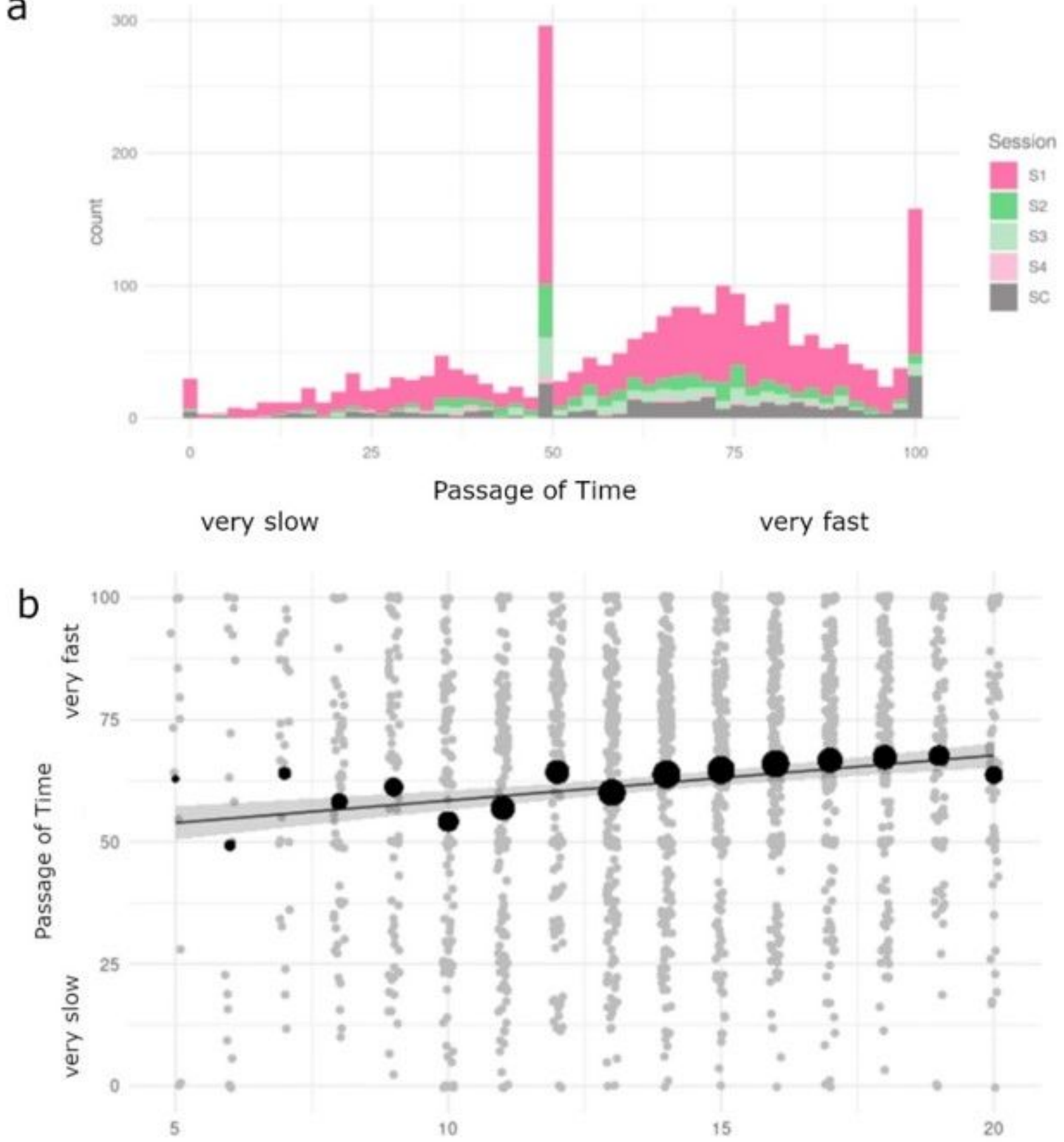

feeling lonely

Subjective Confinement

not feeling lonely

\section{Figure 5}

Passage of Time and subjective confinement. Panel a: Distribution of VAS rating ( 0 to 100$)$ counts for passage of time judgments as a function of session (color coded). Panel $b$ : Passage of time ratings as a function of subjective confinement ( 5 to 20 ). Grey dots are individual data points (per participant, per session, per run). Black dots are the mean passage of time ratings binned by subjective confinement. The 
black line is a regression line estimated from the linear mixed effect model with the $95 \%$ confidence interval (gray shade). The less lonely subjects felt, the faster the passage of time felt.

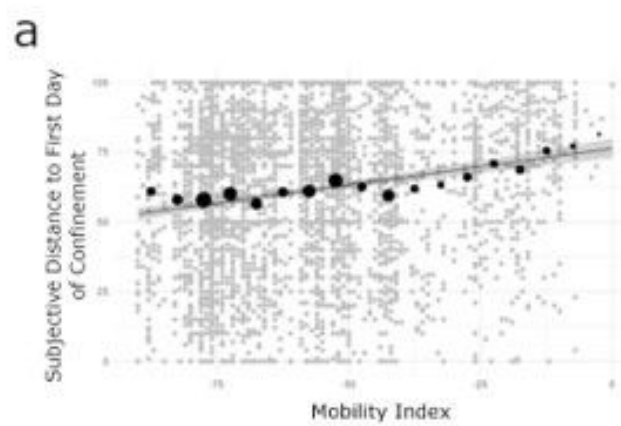

b

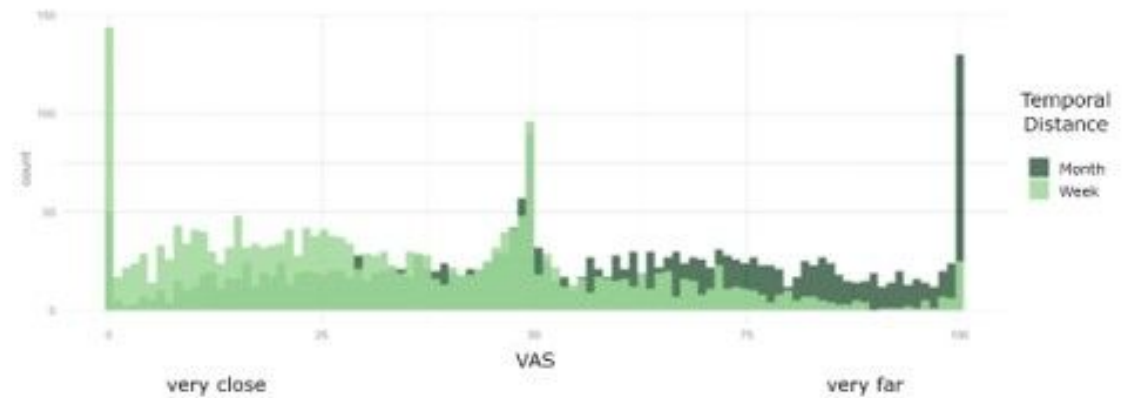

C
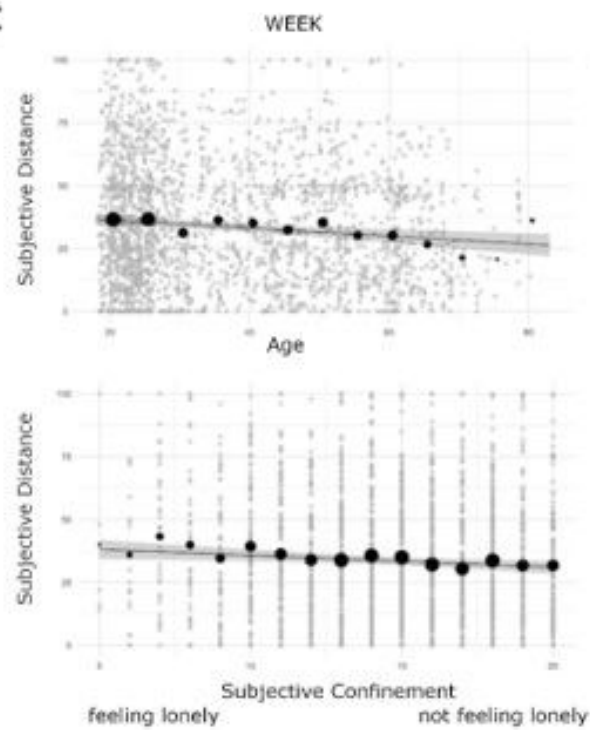
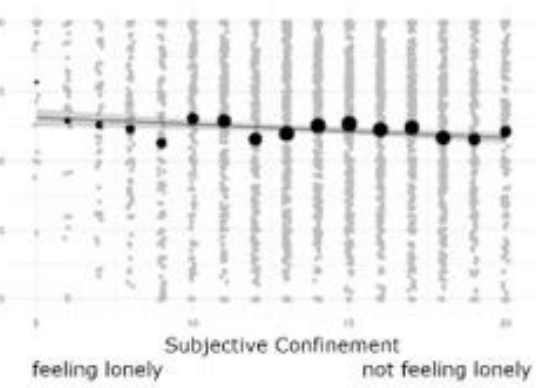

very far
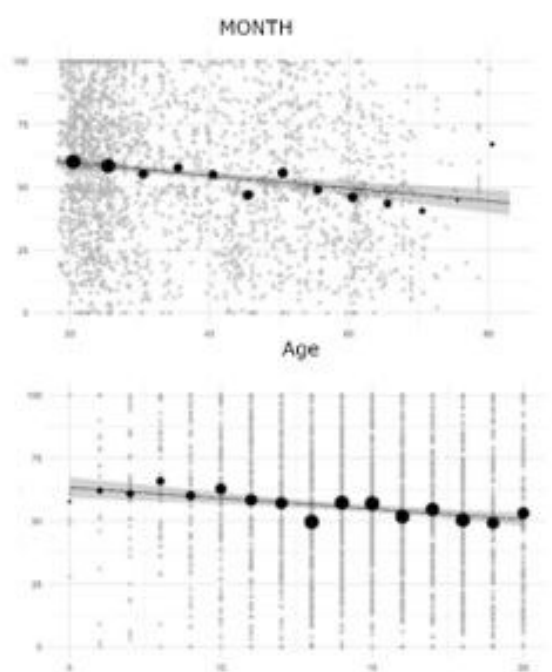

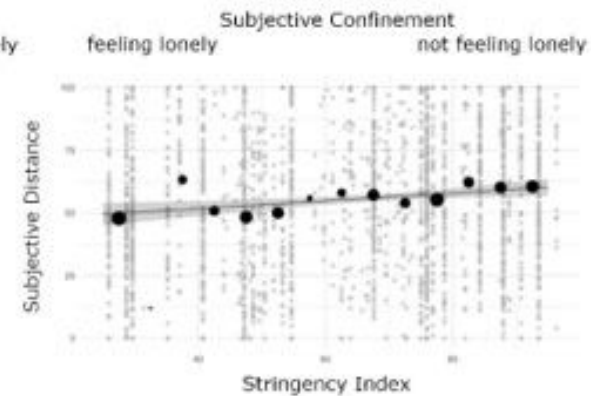

\section{Figure 6}

Subjective temporal distances. Panel a: Subjective temporal distances to the first day of lockdown as a function of the mobility index (left) and the index of subjective confinement. Black dots are the mean subjective temporal distances binned by mobility (left) and subjective confinement (right). Black lines are 
regression lines estimated from the linear regression model with confidence interval (gray shade).Panel b: The distribution of future subjective temporal distances obtained for "next week" (pale green) significantly differed from those obtained for "next month" (dark green; $F(1,3169)=1171.9, p=2.2 e-16$ ). Panel c: Subjective temporal distances to "next week" (left column) and "next month" (right column) as a function of age (top row), subjective confinement index (middle row) and stringency index (bottom). Black dots are the mean subjective temporal distances binned by age (top), subjective confinement (middle), and stringency (bottom). Black lines are regression lines estimated from the linear model with $95 \%$ confidence interval (gray shade).

\section{Supplementary Files}

This is a list of supplementary files associated with this preprint. Click to download.

- Supplementallnformation.docx 